Draft version August 29, 2018

Preprint typeset using $\mathrm{LAT}_{\mathrm{E}} \mathrm{X}$ style emulateapj v. 5/2/11

\title{
THERMAL PROCESSES GOVERNING HOT-JUPITER RADII
}

\author{
DAvid S. Spiegel ${ }^{1,2}$, AdAm Burrows ${ }^{2}$ \\ ${ }^{1}$ Astrophysics Department, Institute for Advanced Study, Princeton, NJ 08540 and \\ ${ }^{2}$ Department of Astrophysical Sciences, Peyton Hall, Princeton University, Princeton, NJ 08544 \\ Draft version August 29, 2018
}

\begin{abstract}
There have been many proposed explanations for the larger-than-expected radii of some transiting hot Jupiters, including either stellar or orbital energy deposition deep in the atmosphere or deep in the interior. In this paper, we explore the important influences on hot-Jupiter radius evolution of $(i)$ additional heat sources in the high atmosphere, the deep atmosphere, and deep in the convective interior; (ii) consistent cooling of the deep interior through the planetary dayside, nightside, and poles; (iii) the degree of heat redistribution to the nightside; and (iv) the presence of an upper atmosphere absorber inferred to produce anomalously hot upper atmospheres and inversions in some close-in giant planets. In particular, we compare the radius expansion effects of atmospheric and deep-interior heating at the same power levels and derive the power required to achieve a given radius increase when night-side cooling is incorporated. We find that models that include consistent day/night cooling are more similar to isotropically irradiated models when there is more heat redistributed from the dayside to the nightside. In addition, we consider the efficacy of ohmic heating in the atmosphere and/or convective interior in inflating hot Jupiters. Among our conclusions are that $(i)$ the most highly irradiated planets cannot stably have $u B \gtrsim 10 \mathrm{~km} \mathrm{~s}^{-1}$. Gauss over a large fraction of their daysides, where $u$ is the zonal wind speed and $\widetilde{B}$ is the dipolar magnetic field strength in the atmosphere, and (ii) that ohmic heating cannot in and of itself lead to a runaway in planet radius.
\end{abstract}

Subject headings: planetary systems - radiative transfer

\section{INTRODUCTION}

The discovery of the "hot Jupiter" class of exoplanets (Mayor \& Queloz 1995; Marcy \& Butler 1996) ushered in a variety of mysteries, of which perhaps the first was how they came to exist, since planetformation models did not generally predict Jupitermass objects in few-day orbit: (Boss 1995; Lissauer 1995). When HD 209458b, the first transiting planet discovered (Henry et al. 2000; Charbonneau et al. 2000 ), was found to have a radius $\gtrsim 30 \%$ larger than Jupiter's, a new mystery was born: why are some hot Jupiters as inflated as they are (Burrows et al. 2000; Fortnev \& Hubbard 2004; Laughlin et al. 2005; Burrows et al. 2007a)? More than a decade later, there were at least 50 transiting planets known with masses greater than a third of Jupiter's and radii greater than 1.3 times Jupiter's The radii of these objects were initially seen as surprising because theoretical evolutionary models suggested that, at the inferred ages of the systems (often more than a billion years old), a hydrogen/helium gas-giant planet "should" have a radius significantly smaller, close to

dave@ias.edu,burrows@astro.princeton.edu,

1 Although, Struve (1952) did suggest the possibility that objects such as what are now called "hot Jupiters" might exist. 2 See http://exoplanet.eu (Schneider et al. 2011), http:/ /exoplanets.org (Wright et al. 2011), or http://exoplanet.hanno-rein.de/ (Rein 2012).
$1.0 R_{J}$, where $R_{J}$ is Jupiter's radius 3 The largest of the hot Jupiters have strikingly large radii, including the 2.04- $R_{J}$ HAT-P-32b (Hartman et al. 2011), the 1.99- $R_{J}$ WASP-17b (Anderson et al.|2011), the 1.83$R_{J}$ HAT-P-33b (Hartman et al. 2011), the 1.74- $R_{J}$ WASP-12b (Chan et al. 2011), and the 1.71- $R_{J}$ TrES4 (Chan et al. 2011). At present, although a number of potential explanations for these planets' large radii have been suggested, it is unclear whether a single "inflating" mechanism predominates.

The processes that have been suggested to explain the surprisingly large radii include both $(i)$ extra power sources in the interior and $(i i)$ enhanced opacity or atmospheric stratification (Burrows et al. 2007a; Baraffe et al. 2010). In addition, there is a subtle distinction between the radius typically calculated in evolutionary cooling models (the radius of the photosphere) and the radius observed in transit; however, this so-called "transit radius effect" (Burrows et al. 2003; Baraffe et al. 2003) increases the apparent radius by no more than $\sim 5 \%$ relative to the radial photosphere. Furthermore, the ("Roche") shape of an object in a strong tidal field can differ from spherical, which has a slight effect on the observed transit radius (Budaj 2011). Explanations of both category $(i)$ and

\footnotetext{
${ }^{3}$ We note that some planets are smaller than expected, which can be explained by the presence of either a heavy-element core or heavy elements throughout the envelope (Guillot et al. 2006; Burrows et al. 2007a).
} 
category $(i i)$ tend to rely on the influence of the star and, therefore, naturally predict that the hottest hot Jupiters (with incident irradiation more than 10,000 times Jupiter's) should have the highest probability of being significantly larger than Jupiter. This prediction is borne out by observations (Laughlin et al. 2011; Demory \& Seager 2011), as can be seen in Fig. 1.

Heating the convective interior of a planet directly increases its bulk entropy and, hence, its radius (Burrows et al. 1994; Bodenheimer et al. 2001; Liu et al. 2008; Spiegel \& Burrows 2012; Marleau \& Cumming 2013). Possible sources of heat in the interior include tidal dissipation of orbital eccentricity (Bodenheimer et al. 2001; Wu 2005a, b; Jackson et al. 2008; Liu et al. 2008; Ibgui \& Burrows 2009; Miller et al. 2009; Leconte et al. 2010; Ibgui et al. 2010, 2011), dissipation of thermal tides (Arras \& Socrates 2009b a, 2010; Socrates 2013), dissipation of downwardly propagating gravity waves (Guillot \& Showman 2002; Showman \& Guillot 2002), and ohmic heating from the dissipation of currents in the partially ionized interior (Batygin \& Stevenson 2010; Batygin et al. 2011; Huang \& Cumming 2012; see also Perna et al. 2010a, who discussed a similar ohmic heating mechanism that might occur in the radiative portion of the atmosphere). Since many hot Jupiters have nearly circular orbits in which there should be little present-day tidal dissipation, steady-state tidal heating probably cannot generically explain all inflated radii. The possibility remains that a planet might have experienced an episode of tidal circularization in the recent past, injecting significant energy, increasing its radius, and effectively "resetting the clock" on its evolutionary shrinkage (Ibgui \& Burrows 2009). However, since the Kelvin-Helmholtz timescale of an inflated hot Jupiter planet is on the order of $\sim 100$ million years, far shorter than the ages of the systems, this sort of explanation requires some fine tuning in the timing and, therefore, in the initial orbital configuration and efficiency of tidal dissipation. While it might explain the radii of some inflated hot Jupiters, it is not a preferred explanation for the entire population (Leconte et al. 2010; Ibgui et al. 2011). Indeed, Ibgui et al. (2011) found via an exhaustive parameter study that the extremely large radius $\left(\sim 1.7 R_{J}\right)$ of TrES-4, for instance, cannot be explained simply by a recent episode of tidal dissipation.

Heating the atmosphere of a planet can puff up its outer few scale heights, but cannot directly explain the radii of many of the larger hot Jupiters (e.g., those with radii $\gtrsim 1.5 R_{J}$ must almost certainly have inflated interiors). However, since the atmosphere of a planet is the conduit through which the convective interior radiatively loses its heat (entropy), the thermal structure of the atmosphere critically mediates the evolution of the thermodynamics of the interior. The intense stellar irradiation that hot Jupiters experience can dramatically change the vertical profiles of their atmospheres, inducing a deep isothermal layer through which evolutionary cool- ing is largely effectively stanched Guillot et al. 1996; Burrows et al. 2000, 2003, 2006, 2007a; Chabrier et al. 2004; Liu et al. 2008; Hansen 2008; Guillot 2010), and significantly modifying the evolution from that of a less strongly irradiated planet (such as those of our solar system; Fortney et al. 2011) or of widely separated planets or brown dwarfs (Burrows et al. 2001; Baraffe et al. 2003; Marlev et al. 2007; Fortnev et al. 2008b; Burrows et al. 2011; Spiegel \& Burrows 2012; Marleau \& Cumming 2013; Paxton et al. 2013). The loss of heat from the interior can be further slowed by increased atmospheric opacity (Burrows et al. 2007a), or by the reduced efficiency of convective heat transport due to double-diffusive convection (Chabrier \& Baraffe 2007; Leconte \& Chabrier 2012). Budaj et al. (2012) examined the influence on atmosphere structure and on planet cooling of a variety of physical effects expected in hot Jupiter atmospheres, including possible extra optical absorbers, the redistribution of day-side heat to the nightside, and more (although they did not calculate the radius evolution). Moreover, extra heating in the atmosphere of a planet, ultimately driven by the irradiation, can reduce the loss of heat from the interior. Sources of extra atmospheric heating include purely hydrodynamic ones (Showman \& Guillot 2002) and magnetohydrodynamic (MHD) ohmic heating due to atmospheric currents in a partially ionized atmosphere (Perna et al. 2010a.b; Menou 2012a; Rauscher \& Menou 2013). The latter process might not only influence the evolutionary cooling of planets, but also help govern their present-day weather patterns and wind speeds, and was suggested simultaneously with, and independently of, the similar mechanism in the interior introduced by Batygin \& Stevenson. Tides might also provide another source of atmospheric heating, since it is currently unknown whether the dissipation of tides can deposit a significant amount of power above the radiative-convective boundary, which delineates, in our parlance, "interior" from "atmosphere" (Lubow et al. 1997; Ogilvie \& Lin 2007; Goodman \& Lackner 2009)

In this paper, we examine the generic character of a variety of potential mechanisms that have been suggested to explain the inflated hot Jupiters. In $\$ 2$, we briefly review observed trends in the dependence of planetary radii and bulk entropy on mass and incident stellar irradiation. In 33, we discuss how atmospheric heating affects evolutionary cooling and, hence, radius. In 44, we discuss how consistently coupling the day and night sides of planets in evolutionary cooling calculations affects the predicted radii given an extra heating luminosity, or the required extra luminosity to match a given radius at a given age. In \$5, we explore the effect of atmospheric ohmic heating, which might be a particularly important mechanism in planets with large-scale magnetic fields and fast winds. We present some details of model atmospheres of a variety of hot Jupiters, including free-electron fraction and conductivity, and examine how these atmospheric model details might depend on gross properties of the planets, 


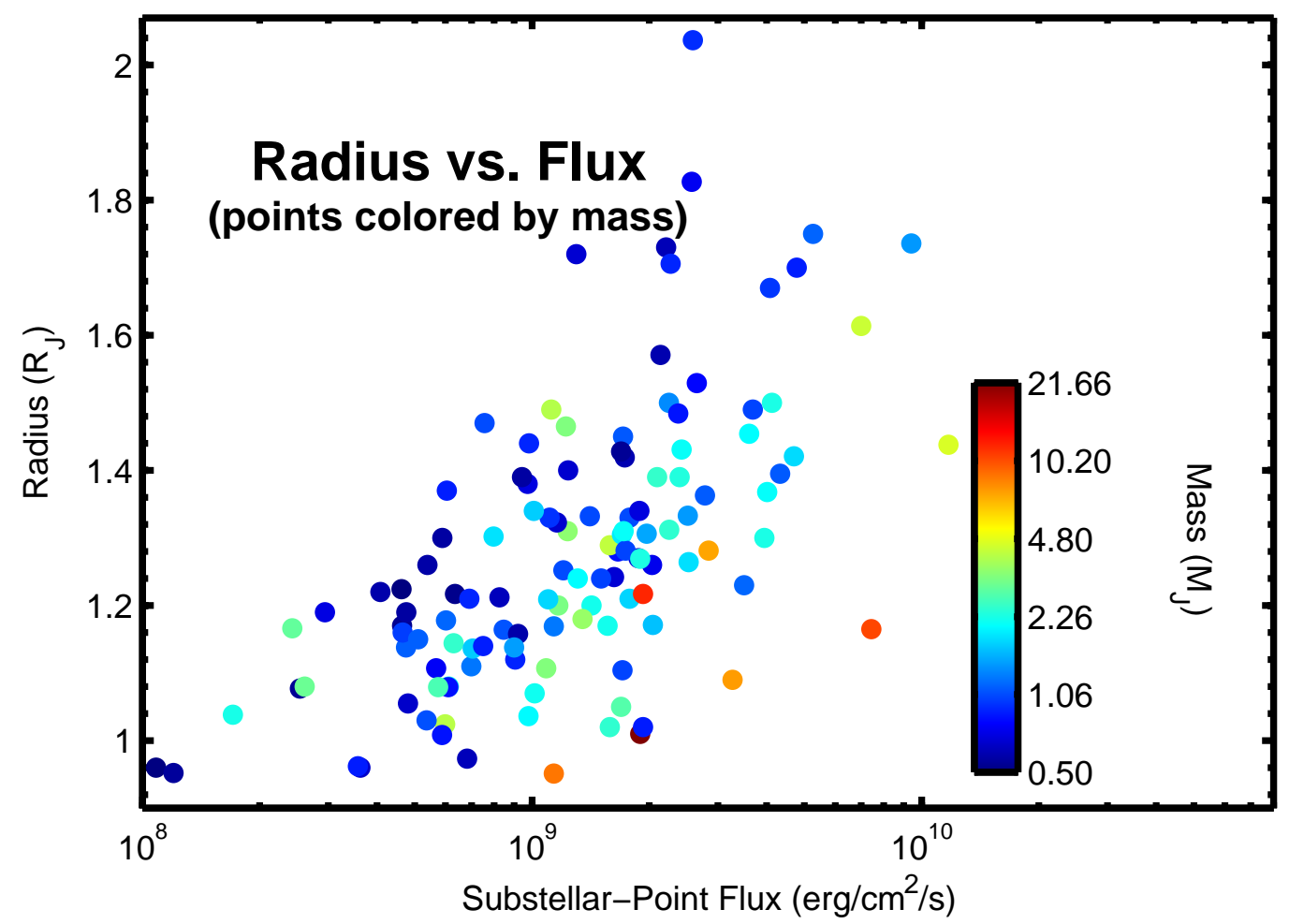

FIG. 1.- Radius vs. incident flux. For each transiting planet in the radius range from 0.9 to $2.07 R_{J}$ found on http://exoplanet.eu (circa February, 2013), we plot the planet's radius against the incident stellar flux at the substellar point; points are colored according to planet mass. The planets with the largest radii tend to be highly irradiated and low mass.

such as surface gravity and incident irradiation. This section concludes by evaluating the stability of planets against a potential runaway inflation process. Finally, we summarize our conclusions in 86

\section{DEPENDENCE OF RADIUS AND ENTROPY ON MASS AND STELLAR FLUX}

It is instructive to examine the relationship between planetary radius and various potentially explanatory variables, such as planet mass and incident stellar flux, among the observed transiting exoplanets. Here, we simply examine the data for all transiting hot Jupiters, making no attempt to correct for selection effects.

Figure 1 presents a scatter plot of planet radius versus incident stellar irradiation. For each transiting planet found on http://exoplanet.eu with radius at least $0.9 R_{J}$, we color the point by the planet's mass. The radii range from our lower cutoff to more than $2 R_{J}$, and the incident fluxes $\left(F_{0}\right.$ values $)$ span more than two decades, from $10^{8} \mathrm{erg} \mathrm{cm}^{-2} \mathrm{~s}^{-1}$ to above $10^{10} \mathrm{erg} \mathrm{cm}^{-2} \mathrm{~s}^{-1}$. The cloud of points exhibit broad scatter, but several trends are evident. Perhaps the most obvious of these is that, even though the color scale is logarithmically spaced in planet mass, there are still far more blue points than red points, indicating that lower-mass planets are significantly more numerous in the sample than higher-mass planets, as has also been seen in some slices of Kepler data (Howard et al. 2012). Furthermore, the planets with the largest radii tend to be highly irradiated and relatively low mass; there are no planets with masses greater than $\sim 5 M_{J}$ and radii greater than $1.29 R_{J}$. The most massive planets do not have radii much above $1.0 R_{J}$. Finally, there is a general upward slope associated with the cloud of points, suggesting that $F_{0}$ could be a contributing factor to explaining planetary radii. Demory \& Seager (2011) noted this trend in $K e$ pler data, as well, finding that incident stellar flux is positively correlated with planet radius at irradiation levels above $\sim 2 \times 10^{8} \mathrm{erg} \mathrm{cm}^{-2} \mathrm{~s}^{-1}$.

Figure 2 shows inferred planetary specific entropy per nucleon plotted against planet radius. For the same sample of planets shown in Fig. 1, we compute the bulk interior specific entropy that corresponds to the listed radius and mass, assuming solar composition and no heavy-element core. Both panels show the same cloud of points; the points are colored in the left panel by mass and in the right panel by incident stellar flux. In the right panel, iso-mass contours are overlaid. The transiting planets appear to occupy a fairly narrow swath of the radius-specific entropy plane, although the true scatter might be slightly different because of variations in bulk composition and core mass.

\section{THE BASIC EFFECT OF ATMOSPHERIC HEATING ON RADIUS}

\subsection{Modeling Evolutionary Cooling}



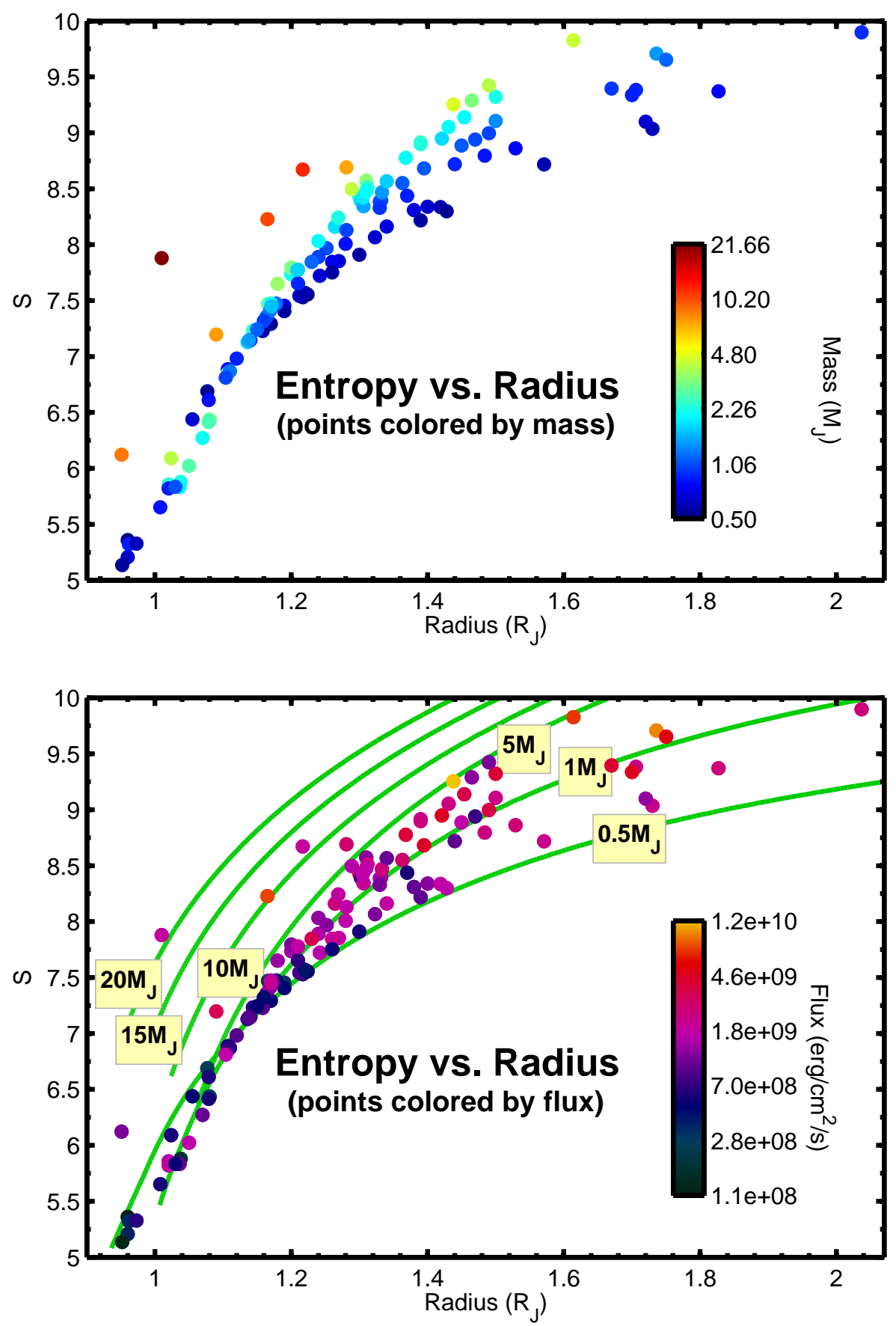

Fig. 2.- Entropy vs. radius, assuming solar composition and no heavy-element core. For each planet in the radius range from 0.9 to $2.07 R_{J}$ on http://exoplanet.eu (in Feburary, 2013), we infer the bulk interior specific entropy that corresponds to the listed radius and mass. Top: The colors of points indicate planet mass. The most massive planets do not have radii much above $1.0 R_{J}$; equivalently, the most inflated planets tend to have lower masses. Bottom: Points are colored according to the incident stellar flux. Contours of constant mass in the radius-specific entropy plane are overlaid as green curves. 
At a given mass, the radius of an isolated $\mathrm{H} / \mathrm{He}$ sphere with a specified helium fraction depends only on its interior entropy (Zapolsky \& Salpeter 1969) 4 At the same mass and specific entropy, the radius can be smaller if there is a heavy element core (Guillot et al. 2006; Burrows et al. 2007a) or if either the helium fraction is larger or there is nonzero metallicity in the bulk interior (Zapolsky \& Salpeter 1969; Spiegel et al. 2011). The thermal state evolves with time as energy flows from the interior to regions from which the optical depth to infinity is low, at which point energy eventually radiates to space, leading to a loss of entropy and a shrinking radius. This evolution depends on the atmosphere, since a higher opacity atmosphere will cause a planet to cool more slowly.

If a planet is not isolated, but rather is irradiated by a nearby star, energy propagates inward as well as outward. In such a situation, the thermal evolution of the interior depends on the net outward radiative flux from the deep interior $\left(F_{\text {net }}\right)$ through the atmosphere. The dayside of a highly irradiated planet tends to have a lower net cooling flux for a given mass and entropy than an isolated planet has. The effective temperature $\left(T_{\text {eff }}\right)$ of an object is the temperature corresponding to the bolometric net outward flux $\left(T_{\text {eff }}=\left\{F_{\text {net }} / \sigma_{\mathrm{SB}}\right\}^{1 / 4}\right.$, where $\sigma_{\mathrm{SB}}$ is the Stefan-Boltzmann constant). Note that the effective temperature, which characterizes the difference between the outgoing and incoming fluxes, should not be confused with the "equilibrium temperature" $T_{\text {eq }}$, which characterizes just the incoming flux and is roughly the temperature of the photospheric region of the atmosphere. In hot Jupiter atmospheres, the approximate flux ratio $\left(T_{\text {eq }} / T_{\text {eff }}\right)^{4}$ can be of order $10^{4}$. In our evolutionary calculations, we establish a mapping between surface gravity $(g)$, specific entropy $(S)$, and effective temperature by precalculating a large grid of one-dimensional, non-gray, radiativeconvective atmosphere models with different effective temperatures and surface gravities and finding the interior adiabat associated with each one, as described in Burrows et al. (1997). One commonly used approach is to take the downward flux in the atmosphere models to be the average (over the planet's surface area) of the irradiating flux, which is $1 / 4$ of the incident flux at the substellar point (this is sometimes described as using a "geometric beaming factor" $f=1 / 4$ - see, e.g., the appendix of Spiegel \& Burrows 2010). We find the function $s\left[T_{\text {eff }}, g\right]$ and invert it to obtain the cooling rate $T_{\mathrm{eff}}[s, g]$. This mapping of $(s, g)$ pairs to $T_{\text {eff }}$ is what we refer to as the "atmospheric boundary condition." (We describe another approach, which couples the cooling through different portions of the atmosphere - e.g., the day and night sides - in 84 .)

\subsection{The Influence of Atmospheric Heating: Extra Absorber}

4 Entropy is a useful diagnostic variable because brown dwarfs and giant planets are fully convective and, hence, at approximately constant specific entropy throughout (Burrows \& Liebert 1993).
The temperature-pressure profile of a planet's atmosphere is inextricably linked to the planet's rate of cooling. Radiative processes that change the vertical thermal structure, therefore, affect the thermal evolution.

A striking feature that has emerged from studies of the emergent radiation from hot Jupiters is that many of these objects appear to have thermal inversions in their upper atmospheres, in which the temperature increases with height above a relative minimum (Hubenv et al. 2003; Burrows et al. 2007b; Knutson et al. 2008; Fortnev et al. 2008a; Spiegel et al. 2009; Madhusudhan \& Seager 2010; Knutson et al. 2010; Madhusudhan 2012). The thermal inversions presumably result from an enhanced opacity in the short wavelength part of the spectrum (optical and ultraviolet), above the alkali metal opacity (mostly sodium and potassium) that some equilibrium chemistry models have suggested might contribute the bulk of the optical opacity in hot Jupiter atmospheres (Sharp \& Burrows 2007). Titanium oxide $(\mathrm{TiO})$ has been suggested as the source of the extra opacity (Hubeny et al. 2003), though it is not clear whether hot Jupiter atmospheres are vigorously enough mixed to bring enough of a heavy, refractory species to the upper atmosphere where it would need to be to cause the inversions (Spiegel et al. 2009; Parmentier et al. 2013). Note that "thermo-resistive" heating as suggested by Menou (2012b) offers a way to heat the upper atmosphere without needing to bring a heavy species to great altitude, although whether it can generically provide sufficient upper-atmosphere power to explain the inferred upper-atmosphere heating remains uncertain.

If all else about a model is held constant, an enhanced optical opacity acts as an anti-greenhouse effect (Hubeny et al. 2003), heating the upper atmosphere and cooling the deeper atmosphere 5 In particular, at fixed $T_{\text {eff }}$, increasing the optical opacity decreases the temperature at depth, in the vicinity of the radiative-convective boundary (see, e.g., Figs. 15 of Spiegel et al. 2009), and, therefore, decreases the entropy of the convective region. However, if we imagine taking a planet that has no thermal inversion and adding an extra optical absorber so as to create a thermal inversion, the interior entropy clearly does not suddenly decrease in response to altered opacity. Instead, in order to keep the interior entropy constant, the effective temperature (i.e., the interior cooling rate) must increase. One might therefore expect that models with an extra optical absorber and a thermal inversion would cool faster than those without these features. Budaj et al. (2012) show that, at fixed surface gravity and interior specific entropy, the effective temperature of a planet increases with the strength of an extra optical absorber. Here, we calculate the influence that this process has on thermal and

\footnotetext{
5 The greenhouse effect, in contrast, results from enhanced infrared opacity and heats the deeper atmosphere while cooling the upper atmosphere.
} 
radius evolution.

Figure 3 displays how one-dimensional models indeed exhibit this behavior. The top panel of Fig. 3 shows the influence of adding an extra optical absorber. At fixed $T_{\text {eff }}$, the deep atmosphere is cooled relative to an atmosphere without the extra absorber (represented as $\kappa^{\prime}$ in the figure). If the effective temperature is allowed to vary so as to maintain fixed interior specific entropy, the model with the extra absorber achieves an effective temperature nearly $20 \%$ greater $(215 \mathrm{~K}$ vs. $180 \mathrm{~K})$, implying a net cooling rate more than twice as great. This increased cooling rate is clear in the right panel of Fig. 3, which shows the evolution of radius for 1-D model planets with and without an extra absorber. The model with the extra absorber shrinks more rapidly[

\subsection{The Influence of Atmospheric Heating: Below the Photosphere}

Anything that modifies the radiative processes of the atmosphere can affect evolutionary cooling. In addition to changes in opacity, as discussed in 33.2 above, there are other ways that atmospheres can change the mapping of $S$ and $g$ to $T_{\text {eff }}$. Adding heat to the atmosphere can affect the interior entropy and, therefore, the radius. Consider if some fraction $\varepsilon$ of the intercepted radiation is converted to another form of energy (say, mechanical energy) that then dissipates as heat below the optical photosphere. At a given mass, effective temperature, and incident irradiation, a planet where $\varepsilon$ is greater will have an atmosphere that matches to a higher entropy adiabat in the convective region, and, therefore, that corresponds to a higher entropy (and larger radius).

Figure 4 illustrates how a power source in the radiative part of a planet's atmosphere can modify the planet's bulk entropy (and, hence, the radius) at a given effective temperature. In this figure, a range of model planets is displayed, all of which have the surface gravity and incident stellar irradiation of HD 209458b, and all have an internal flux temperature of $T_{\text {eff }}=180 \mathrm{~K}$. The atmosphere models are calculated with the atmosphere radiative transfer code COOLTLUSTY (Hubenv 1988; Hubenv \& Lanz 1995; Hubenv et al. 2003; (Burrows et al. 2006), and represent solutions to the radiative transfer equation in which we enforce radiative and chemical equilibrium.

In the model planets shown in Fig. 4, a small fraction of the incident irradiation is deposited at various depths in the atmosphere, with the power spread in a Gaussian distribution over a region of width 0.5 in log pressure. The models differ according to the fraction of incident irradiation that is deposited at depth in the atmosphere (shown on the abscissa) and according to where in the atmosphere the power is deposited (shown as different colored curves). When the power is

\footnotetext{
6 However, the effect of an extra optical absorber almost entirely disappears in the context of a " $1+1$ "-dimensional model, as discussed in 4 below.
}

deposited high in the atmosphere (at pressures below $\sim 1$ bar), it has little influence on the interior adiabat and, therefore, little influence on the radius. When power is deposited deeper, it can have a significant influence on the interior adiabat's entropy, and a correspondingly large influence on a planet's radius (at fixed $T_{\text {eff }}=180 \mathrm{~K}$, with these effects both being larger when more power is deposited). The black dashed line shows the radius $\left(\approx 1.25 R_{J}\right)$ that this model planet has with no extra atmospheric heating. Depositing $1 \%$ of the incident power at 10 bars means that a planet with $T_{\text {eff }}=180 \mathrm{~K}$ has a radius $\sim 5 \%$ larger (which corresponds to an increment in specific entropy of $\sim 0.3 k_{B}$ baryon $^{-1}$, where $k_{B}$ is Boltzmann's constant. If the power is deposited at 30 bars, the planet has a radius nearly $20 \%$ larger, and if the power is deposited at 100 bars, the radius is nearly $1.9 R_{J}$.

Holding $T_{\text {eff }}$ fixed, however, means that the different models in Fig. 4 would correspond to different ages in a true evolutionary calculation, with the larger radius models corresponding to younger planets. This figure demonstrates the general character of the effect of atmospheric heating on radius, but a more useful comparison would be to hold constant, not $T_{\text {eff }}$, but rather age. It turns out that in the context of 1-D models, an extra power source in the deep atmosphere can result in a dramatically reduced cooling rate and, hence, significantly inflated radii. This effect is muted when night-side cooling is taken into account (see \$4).

\section{EVOLUTIONARY COOLING WITH CONSISTENT DAY/NIGHT COUPLING}

\subsection{The Influence of Non-Isotropic Heating/Cooling}

A gas giant's evolutionary cooling and shrinking are mediated by details of the three-dimensional atmospheric structure. Isolated (or nearly isolated) objects, such as wide-separation planets or brown dwarfs, may, to a reasonable degree of approximation, be treated as one dimensional (i.e., as spherically symmetric). Hot Jupiters, on the other hand, are expected to be tidally locked to their stars (although see Arras \& Socrates 2010) and, therefore, to experience a strong, permanent asymmetry in irradiation between the dayside and the nightside. As described in 3.1 . treatments in the literature of the thermal evolution of hot Jupiters have often used one-dimensional models in which the objects are taken to be irradiated by the global average irradiation, $(1 / 4) F_{0}$, where $F_{0}$ is the substellar point flux (the "stellar constant") for the planet (Guillot et al. 1996; Burrows et al. 2000; Bodenheimer et al. 2001; Burrows et al. 2003; Baraffe et al.|2003; Bodenheimer et al.|2003; Gu et al. 2003; Burrows et al. 2004; Fortnev \& Hubbard 2004; Baraffe et al. 2004; Chabrier et al. 2004; Laughlin et al. 2005; Baraffe et al. 2005, 2006; Burrows et al. 2007a; Fortney \& Marley 2007; Marley et al. 2007; Chabrier \& Baraffe 2007; Liu et al. 2008; Baraffe et al. 2008; Ibgui \& Burrows 2009; Miller et al. 2009; Leconte et al. 2010; Ibgui et al. 2010, 2011). If horizontal variations 

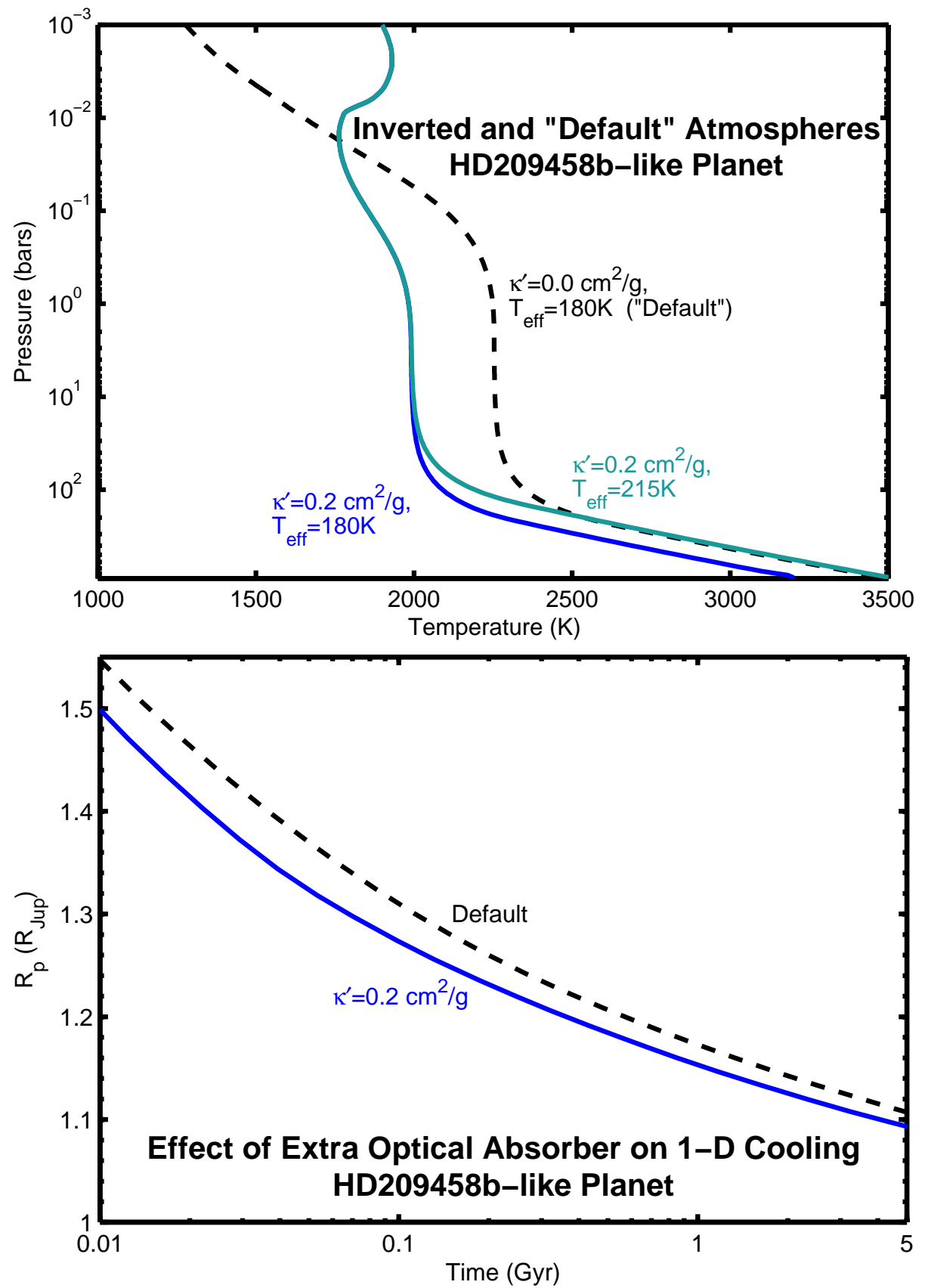

FIG. 3.- Atmosphere and evolution models of HD 209458b-like planet, with and without extra optical absorber. Top: Comparison of atmospheres at constant $g$ and constant $T_{\text {eff }}$ (black and blue) or constant interior entropy (black and aqua): These atmosphere models all have $\log _{10} g=3.0$ where $g$ is measured in cgs units (similar to HD 209458b). At constant $T_{\text {eff }}$ (dashed black curve and blue curve have $\left.T_{\text {eff }}=180 \mathrm{~K}\right)$, an extra optical absorber $\left(\kappa^{\prime}-\right.$ see, e.g., [Spiegel et al. 2009) heats the upper atmosphere and cools the lower atmosphere. At constant interior specific entropy (dashed black curve and aqua curve have the same interior adiabat specific entropy), the extra optical absorber increases $T_{\text {eff }}$ by $\sim 20 \%$ and, therefore, doubles the net cooling luminosity. Bottom: Comparison of radius evolution at constant planet mass: Two radius evolution trajectories are shown, both of which correspond to model planets with a mass of $0.69 M_{J}$, where $M_{J}=1.899 \times 10^{30} \mathrm{~g}$ is the mass of Jupiter. Comparing the two radius evolution curves shows that, as expected, in a 1-D cooling model, the planet with an extra optical absorber and a thermal inversion (blue curve) cools faster and its radius shrinks faster than the planet with a "default" (noninverted) atmosphere (black dashed curve). 
in cooling are large, however, these one-dimensional models might not accurately capture the character of cooling on a highly-irradiated, tidally-locked planet (Guillot \& Showman 2002; Budai et al. 2012).

Hot Jupiters are presumed to be tidally locked in synchronous rotation, with one side in permanent day and the other in permanent night. The dayside has strong static stability imposed by the steady injection of energy (and entropy) high in the atmosphere. The radiative-convective boundary ( $\mathrm{RCB}$ ) extends deeper into the planet as the object cools and shrinks, and by an age of $10^{9}$ yrs or more the statically stable region on the dayside can extend quite deeply into the atmosphere, with a RCB at pressures of hundreds of bars to a kilobar or more (Burrows et al. 2003). The nightside, with no irradiation, might have a RCB at a much lower pressure (by several orders of magnitude), and, therefore, cool more similarly to an isolated object. We note that some dynamical atmospheric circulation models (e.g., Cooper \& Showman 2005) find that day-side and night-side temperatures are fairly similar by a pressure of $\sim 10$ bars, although the poles are significantly cooler. Whether the relatively cool, less statically stable region is on the nightside or at the poles is not terribly important; either deviation from an isotropically cooling planet can cause large changes to the cooling rate.

One may, therefore, significantly improve upon the models typically used, by appropriately coupling simple models of the dayside and nightside together, at consistent interior entropy and surface gravity (Guillot \& Showman 2002; Budaj et al. 2012). Instead of treating the entire atmosphere as though it were uniformly irradiated with the planetary average irradiation, one may produce a "1+1-dimensional" model. First, compute a grid of day-side models, defining a function $T_{\mathrm{eff}}^{\text {day }}[s, g]$. Then, compute a similar grid of night-side models, defining a function $T_{\mathrm{eff}}^{\text {night }}[s, g]$. The total cooling, then, is the sum of the cooling through the dayside and of that through the nightside:

$$
T_{\text {eff }}[s, g]^{4}=\frac{T_{\mathrm{eff}}^{\mathrm{day}}[s, g]^{4}+T_{\mathrm{eff}}^{\mathrm{night}}[s, g]^{4}}{2},
$$

and $T_{\text {eff }}[s, g]$ is given by the fourth root of the above expression. In equation (11), each function must be evaluated at the same $s$ and $g$ : the same $s$ because convection homogenizes specific entropy, and the same $g$ because the planet is (very nearly) spherical. The primary effect of strong irradiation on the dayside is to create a deep, nearly isothermal layer, through which the net flux is nearly zero, thereby strongly decreasing evolutionary cooling (i.e., lowering $\left.T_{\text {eff }}^{\text {day }}\right)$. The nightside and poles lack the dayside's strong irradiation, might remain fully convective out to much lower pressure levels (bars to tens of bars), and, thus, might more efficiently transport heat from the interior to where it may radiate away to space. As a result, at equivalent $s$ and $g$, the night-side cooling or polar cooling is generally much greater than the day-side cooling and, therefore, $T_{\text {eff }}[s, g]^{4} \sim(1 / 2) T_{\text {eff }}^{\text {night }}[s, g]^{4}$. This can be a large effect that significantly increases the net cooling relative to what would be found for a hypothetical isotropically irradiated planet.

The process of horizontal redistribution of day-side atmospheric heat in hot Jupiter atmospheres has been treated in a number of ways in the literature (see the Appendix of Spiegel \& Burrows 2010 for a review; see also Cowan \& Agol 2011 and Madhusudhan \& Seager 2009 ). The most physically motivated $1-D$ parameterization of redistribution is the $P_{n}$ formalism introduced by Burrows et al. (2006) and Burrows et al. (2008), in which a fraction $P_{n}$ ("portion-to-night") of day-side irradiation is removed from the day-side atmosphere and deposited on the nightside in a specified pressure interval (here, $\sim 0.01-0.1$ bars, although the redistribution could in principle happen at other pressure ranges). $P_{n}$ plausibly ranges between 0 (corresponding to no redistribution) and 0.5 (corresponding to a fully mixed planet where the nightside receives half the total intercepted power). In our models, we use $P_{n}$ to represent redistribution, removing day-side flux and adding heat to the nightside.

Figure 5 shows the evolution of the radius of HD 209458b-like models with various atmospheric boundary conditions. Of the seven evolutionary trajectories presented, four represent isotropic (1-D) models, and three represent day/night-merged (1+1D) models. Two curves are shown that also appear in Fig. 3, the "default" 1-D model, shown as a black dashed line; and the $\kappa^{\prime}=0.2 \mathrm{~cm}^{2} \mathrm{~g}^{-1}$ model with a thermally inverted layer created by the extra absorber, shown as a blue curve. As previously noted, the effect of the extra absorber is to increase the cooling rate and reduce the planetary radius at a given age.

However, when we consider the effect of an extra absorber in a $1+1-\mathrm{D}$ model with night-side cooling, the influence of nonzero $\kappa^{\prime}$ nearly disappears. The day/night-merged models in Fig. [5] are bounded by gray dashed-dotted curves that indicate extreme (and unrealizable) atmosphere conditions. These cases consist of isotropic conditions corresponding to day-side only (upper curve) or night-side only (lower curve) atmospheric conditions. The night-side only object has zero irradiation and is significantly heated only by the redistributed heat from the dayside, in this case corresponding to $30 \%$ of the incident irradiation (the heating from below corresponding to the net cooling flux of the object pales in comparison with the redistributed heating from the dayside). Since real planets do not experience isotropic day-side or isotropic nightside conditions, the top and bottom curves simply illustrate bounding cases for the possible range of evolutionary tracks for combinations of irradiated and nonirradiated boundary conditions (with $P_{n}=0.3$ ). The red and magenta curves show $1+1-\mathrm{D}$ evolution models for $P_{n}=0.3$ and $P_{n}=0.1$, respectively. The green curve represents a $1+1-\mathrm{D}$ model for $\kappa^{\prime}=0.2 \mathrm{~cm}^{2} \mathrm{~g}^{-1}$ and $P_{n}=0.3$. Importantly, the red, magenta, blue, and green curves are nearly indistinguishable after a 

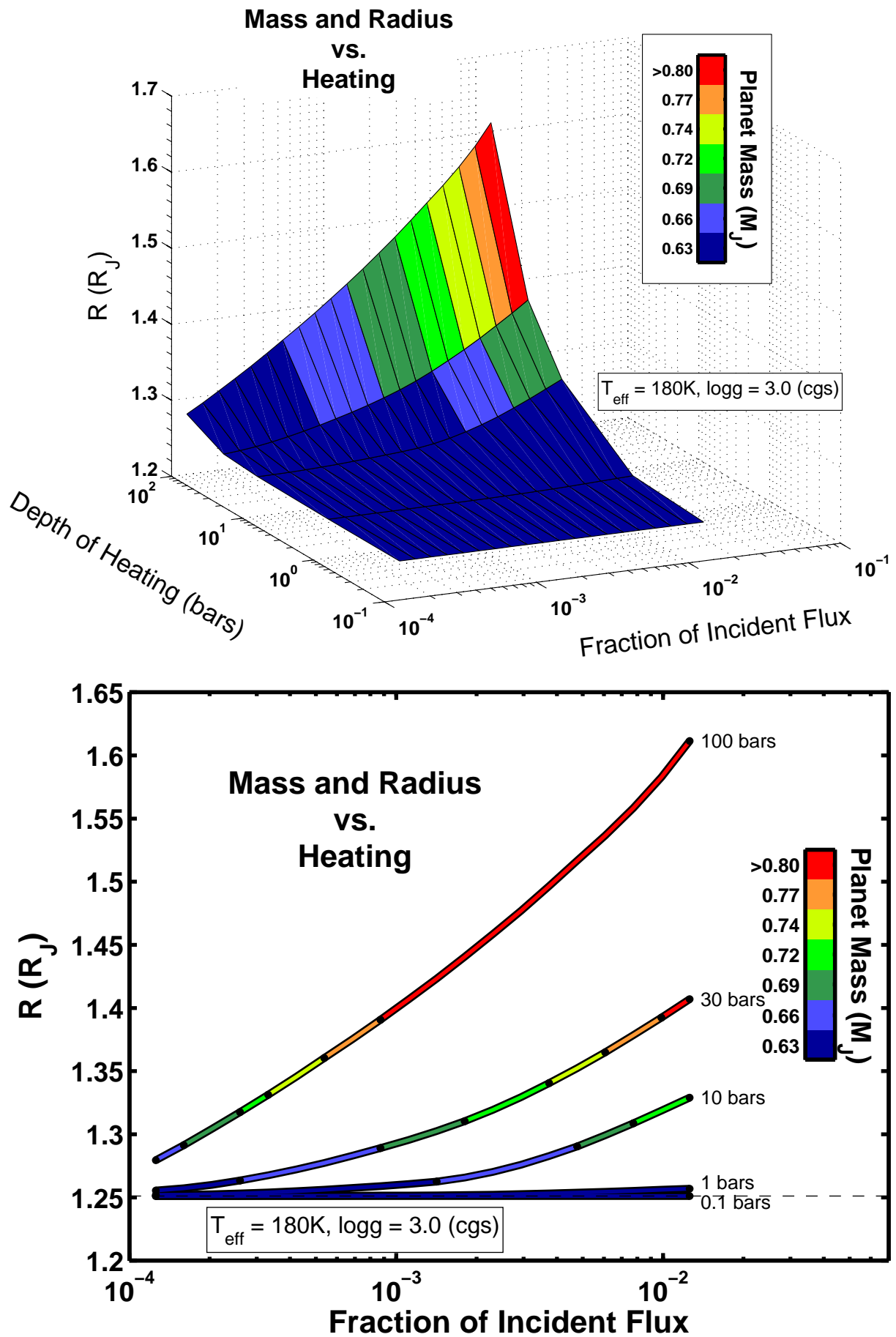

Fig. 4.- Heating at different pressure levels, at constant $T_{\text {eff }}$. Each of the model planets shown here has the surface gravity $\left(1.0 \times 10^{3}\right.$ dyne $\left.\mathrm{g}^{-1}\right)$ and incident irradiation of $\mathrm{HD} 209458 \mathrm{~b}$, and has $T_{\text {eff }}=180 \mathrm{~K}$. In each model, a small fraction of the incident irradiation (fraction shown by the abscissa) is deposited at various depths in the radiative portion of the atmosphere, and is spread over a range of pressures of $\log _{10}$ width 0.5. Top: Planet radius (vertical axis) and mass (color) are shown as a function of the extra power added to the atmosphere and the depth at which it is deposited. At fixed $T_{\text {eff }}$ and $g$, greater power and greater depth in the atmosphere correspond to larger radius and larger mass. Bottom: The same information as is in the top panel is depicted in a different format. Each curve corresponds to a different depth at which power is deposited. At 100 bars, even a very small fraction $\left(10^{-4}\right)$ of incident power corresponds to an increase of a few percent in radius (at constant $T_{r m e f f}$ and $g$ ). In contrast, if the power is deposited at 1 bar, there is hardly any influence on radius even if two orders of magnitude more power is injected. The upshot is that greater the atmospheric heating, and the greater the depth at which the power is deposited, the higher the entropy of the interior adiabat and, therefore, the greater the radius (and, at fixed $g$, the higher the planet mass). The dashed black line shows the specific entropy and the radius with no extra power. 
few tens of Myr. In other words, when night-side cooling is incorporated, all 1+1-D models in Fig. 5 cool faster than the default model, and subtleties such as, in this context, the presence of an optical extra absorber become insignificant.

\subsection{The Influence of Atmospheric vs. Deep Interior Heating}

Various mechanisms might deposit at depth an amount of power that is small in comparison with the irradiating power. This extra heating might occur deep in the atmosphere, at pressures of bars to hundreds of bars, or it might occur in the deep convective interior.

Figure 6] shows radius evolution models of an HD 209458b-like planet with and without either deepinterior or atmospheric heating. Three models demonstrate the influence of extra isotropic atmospheric heating set to $1 \%$ of the incident irradiation (brown curves), in which the extra atmospheric power is deposited in a Gaussian distribution of width 0.5 in log pressure, centered on 10 bars (thin curve), 30 bars (medium curve), and 100 bars (thick curve). Note that when extra heating is at a depth of tens of bars or more, it acts to retard evolutionary cooling, in contrast to the effect of $\kappa^{\prime}$ - which is essentially extra heating at millibars or higher - which acts to accelerate cooling and shrinking. Differences in radius between different models generally (but not always) shrink with time, but after $1 \mathrm{Gyr}$, the model in which $1 \%$ of incident irradiation is deposited at 10 bars is $\sim 0.1 R_{J}$ larger than the default model; the model with extra power at 30 bars is more than $0.2 R_{J}$ larger than the default model after $1 \mathrm{Gyr}$; and the model with extra power at 100 bars is more than $0.5 R_{J}$ larger than the default model after $1 \mathrm{Gyr}$, and has a radius larger than $1.7 R_{J}$ at this age. This is consistent with the findings of Guillot \& Showman (2002), who argued that depositing $1 \%$ of the incident irradiation deep in the atmosphere could have an important radius-inflating effect.

Models in which the dayside's extra atmospheric heating is merged with night-side cooling exhibit a significantly reduced influence of extra day-side heating. This coupled day/night cooling scenario with day-side heating is represented (for the case of heating centered at 100 bars) by the purple curve. If the nightside is unheated, the extra heating on the dayside, which results in such dramatically increased radii in the 1-D cases, has essentially no influence on the radius in the $1+1$-D case (note the very small difference between the 1+1-D models with no day-side heating and with day-side heating, but no night-side heating — the red and purple curves, respectively).

Figure 6 also presents two examples of radius evolution with deep-interior heating - one for a 1-D model (green) and one for a 1+1-D model (aqua). The interior heating in both models is set to $1 \%$ of the incident irradiating power and is assumed to occur in the center of the planet. Note that an extra power source that is deep in the envelope, but not in the center, might have a complicated influence on the thermal structure of the object. Instead of resulting in uniformly higher specific entropy in the deep interior, such an extra power source might inhibit convection (Wu \& Lithwick 2013). We avoid this complexity by assuming that the extra heat is deposited in the center, but we note that fully exploring the structural influence of deep envelope heating is a subject ripe for future work. At early times, the brown curves (1D models with atmospheric heating) have larger radii than the models with an equivalent amount of central heating. However, the models with deep-interior heating reach an inflated asymptotic radius because in such a model the effective temperatures has a strict (positive-definite) floor corresponding to the flux implied by the power source. In contrast, the asymptotic radius of a model with extra atmospheric heating is the zero-temperature, fully degenerate radius approximately $1 R_{J}$ for objects of roughly Jupiter's mass - because the extra heating simply reduces the net cooling rate to a lower, but still nonzero, value. The asymptotic radius of the 1-D model with central heating is roughly $\sim 0.08 R_{J}$ larger than that of the $1+1$-D model with central heating and $P_{n}=0.3$.

Figure 7 depicts the radius evolution of models of an HD 209458b-like planet with a range of levels of central heating power. This figure particularly highlights the contrast between 1-D models (green) and $1+1$-D models with $P_{n}=0.3$ (aqua). For both the isotropic model and the one with night-side cooling, increasing the interior heating power (measured in units of the incident power) from $0.5 \%$ (thin curve) to $1 \%$ (medium curve) results in nearly a $0.1 R_{J}$ increase in asymptotic radius, and increasing the power from $1 \%$ to $2 \%$ (thick curve) results in another $\sim 0.1 R_{J}$ increase. For this set of models, including nightside cooling with $P_{n}=0.3$ results in a decrement of $\sim 0.1 R_{J}$, such that, in the set of models displayed in Fig. 7, achieving the same asymptotic radius requires twice as much central heating power in the context of a $1+1$-D model with $P_{n}=0.3$ as with a $1-D$ isotropic model.

\subsection{The Influence of Redistributive Winds $\left(P_{n}\right)$}

As described in 4.1 in the absence of winds that redistribute energy from the dayside of a planet to its nightside, the nightside would be much cooler than the dayside. Day-night temperature differences, however, lead to pressure gradients that drive redistributive winds (see, e.g., Fig. 3 of Burrows et al. 2010).

Figure 8 demonstrates both the effect of incorporating consistent night-side cooling in an atmosphere model and the influence of the $P_{n}$ redistribution parameter. For variety, in this figure we show the evolution of a model with the mass and irradiation of WASP-12b (Hebb et al. 2009) instead of HD 209458b. The model planet is evolved with three different atmospheric boundary conditions and with the extra interior power that is necessary, according to Ibgui et al. 


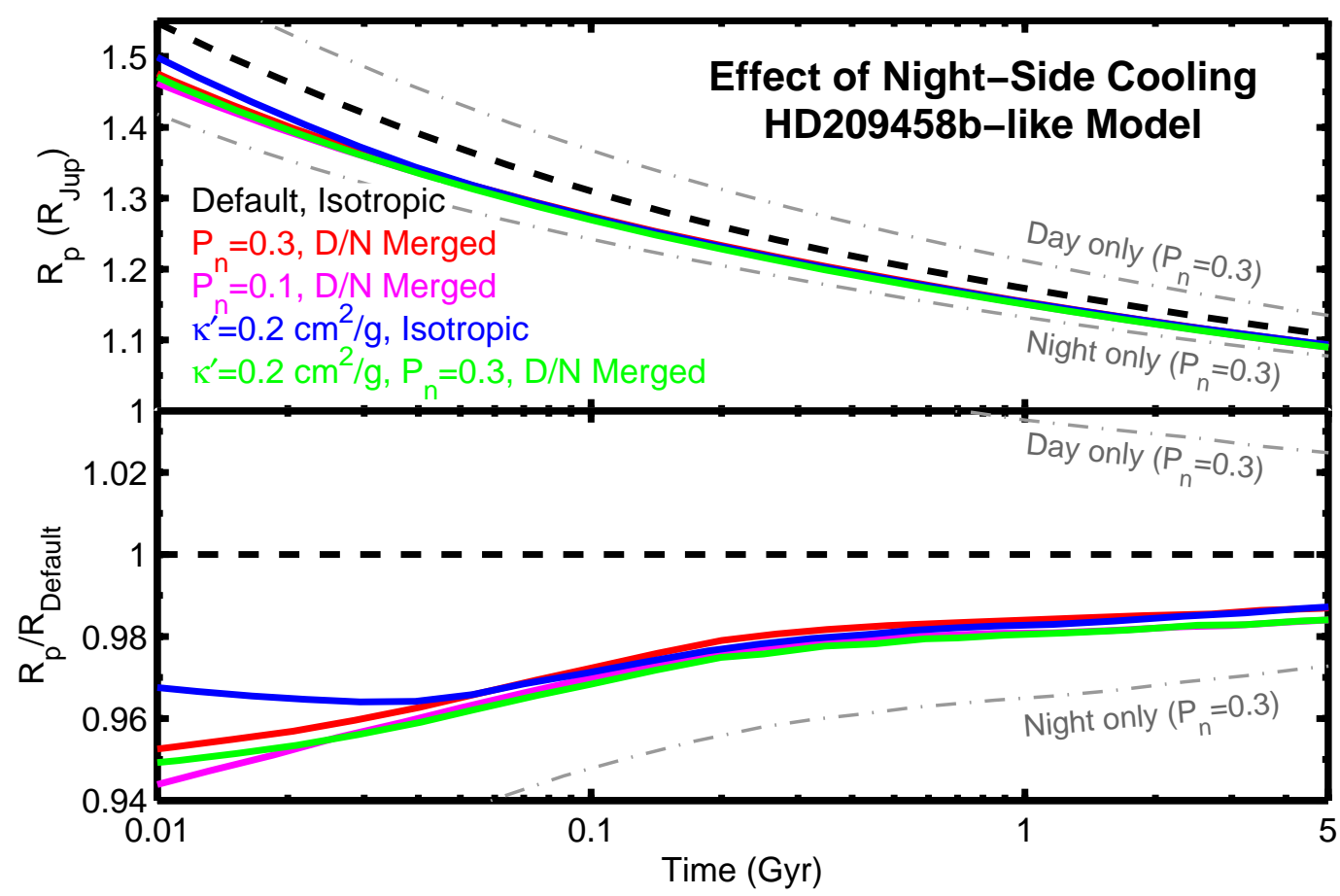

FIG. 5.- Evolution models of an HD 209458b-like planet with and without night-side cooling. Seven radius evolutionary tracks are shown of a planet the mass of HD 209458b $\left(0.69 M_{J}\right)$, five of which are represented with thick lines, corresponding to a 1-D model (isotropic irradiation - black dashed curve), a 1+1-D model with $P_{n}=0.3$ (red curve), a 1+1-D model with $P_{n}=0.1$ (magenta curve), a 1-D model with $\kappa^{\prime}=0.2 \mathrm{~cm}^{2} \mathrm{~g}^{-1}$ (blue curve), and a 1+1-D model with $\kappa^{\prime}=0.2 \mathrm{~cm}^{2} \mathrm{~g}^{-1}$ and $P_{n}=0.3$ (green curve). (The models described as " $\mathrm{D} / \mathrm{N}$ Merged" are the 1+1-D models with day-side and night-side boundary conditions merged consistently.) For illustrative purposes, we include thin gray dashed-dotted lines that indicate evolutionary trajectories for hypothetical planets with boundary conditions corresponding to day-side only and night-side only boundary conditions (i.e., all $4 \pi$ steradians are bounded by either the dayside or the nightside). Real planets clearly do not experience isotropic day-side or isotropic night-side conditions The top and bottom curves represent extreme cases that bound the possible range of evolutionary tracks for mergers of irradiated and non-irradiated boundary conditions (with $P_{n}=0.3$ ). Top: The evolution of the seven models is shown measured in Jupiter radii. Bottom: The evolution of the same models is shown in units of the radius of the "default" model of the same age.

(2010), to maintain its current radius at $\sim 1 \frac{3}{4} R_{J}$ $\left(2.4 \times 10^{-6} L_{\odot}\right.$, or $0.2 \%$ of the incident power $)$. As above, the source of this extra interior power is not specified in this model, but it could correspond to tidal heating or to ohmic heating in the interior. The three boundary conditions correspond to a default (isotropic) 1-D model $(f=1 / 4)$, and two $1+1-\mathrm{D}$ models (with redistribution given by $P_{n}=0.1$ and $\left.P_{n}=0.3\right)$ that couple day-side cooling to night-side cooling in accordance with Eq. (11). As noted by Budaj et al. (2012), redistribution of heat from the day to the night has the dual effects of cooling the dayside and heating the nightside. The former effect slightly increases the dayside's cooling rate, but the latter effect significantly slows night-side cooling. The influences of these two effects on radius evolution have opposite sign, but the (radius-boosting) effect of a warmer nightside is quantitatively much more significant than the (radius-shrinking) effect of a cooler dayside. As a result, the $1+1-\mathrm{D}$ models with consistent day/night cooling cool more rapidly than does the 1-D isotropic model, and the model with $P_{n}=0.3$ asymptotes to a radius more than $0.1 R_{J}$ smaller than the model with isotropic conditions 7 The isotropic

7 This same effect was seen previously in Figs. [5 7 for model, unsurprisingly, asymptotes at the same radius as the analogous model from Ibgui et al. (2010) with identical interior luminosity. The model with $P_{n}=0.3$ has a warmer nightside than the one with $P_{n}=0.1$, which leads to a lower net flux (i.e., a lower effective temperature). Therefore, since the model planet's net cooling is dominated by the nightside's effective temperature, the $P_{n}=0.3$ model asymptotes at a larger radius (by $0.03 R_{J}$ ) than the $P_{n}=0.1$ model.

\section{THE EFFECT OF OHMIC HEATING}

\subsection{Heating in the Atmosphere}

The temperatures and densities that are present in the day-side atmospheres of highly irradiated planets (Fortney et al. 2008a; Spiegel et al. 2009; Madhusudhan \& Seager 2010; Spiegel \& Burrows 2010) imply a small, but nonnegligible, free electron fraction from the partial ionization of species with low ionization potential (mostly atomic potassium and sodium). During the last decade, a wide variety of circulation models have predicted that, near the equator and at depths comparable to the optical and infrared photospheres, hot Jupiters should have eastward (prograde) wind velocities $(\mathbf{u})$ of $\sim 1 \mathrm{~km} \mathrm{~s}^{-1}$ or 


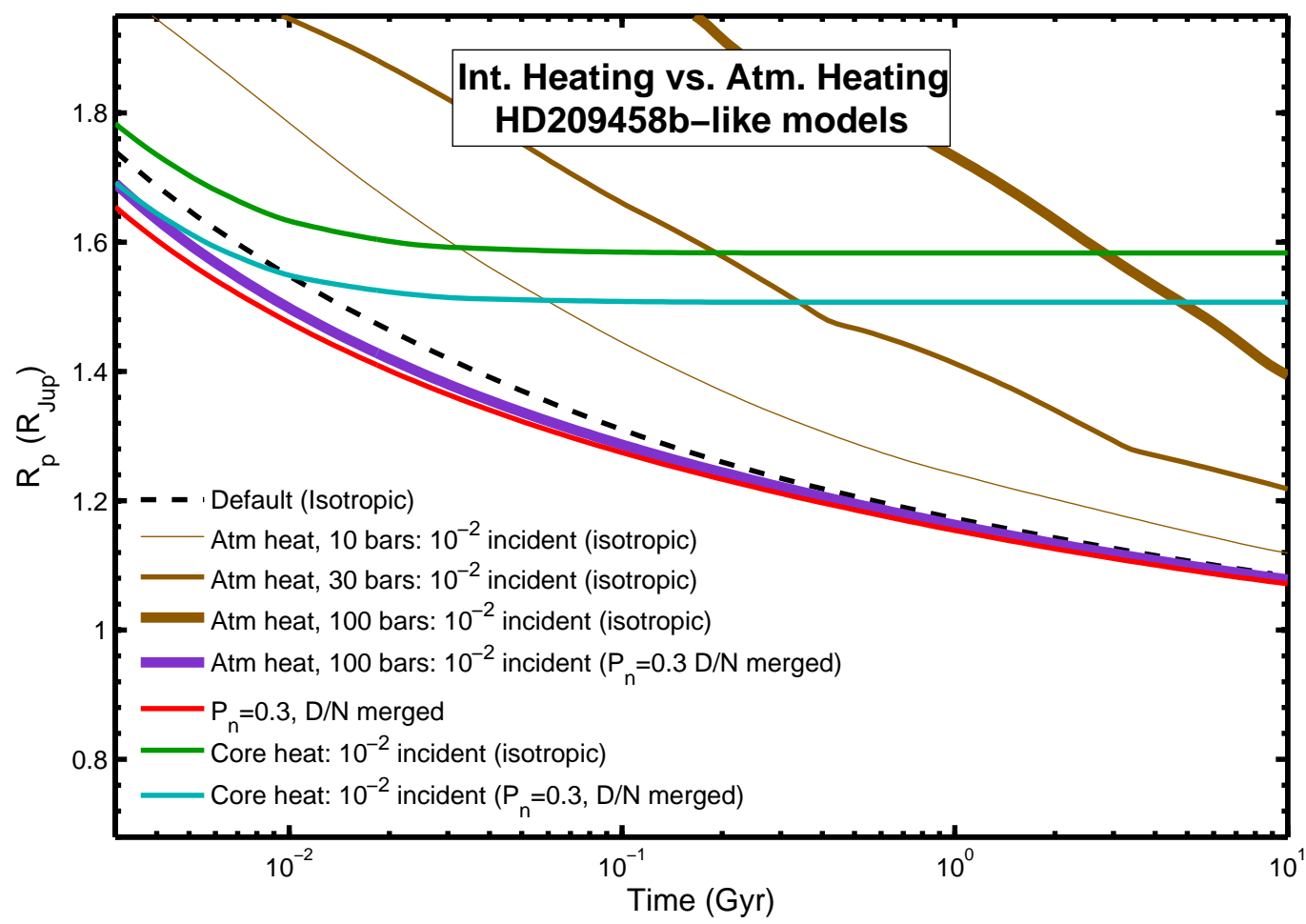

FIG. 6.- Evolution models of an HD 209458b-like planet, with and without deep-interior or atmospheric heating. Eight models are presented: the default (isotropic heating) model (black dashed); three 1-D models with extra atmospheric heating set to $1 \%$ of the incident irradiation, deposited at 10 bars (thin brown), 30 bars (medium brown), and 100 bars (thick brown); a 1+1-D model with $P_{n}=0.3$ and with extra heating deposited at 100 bars and an unheated nightside (thick purple); a 1+1-D model with no extra heating and $P_{n}=0.3$ (red); a 1-D model with deep-interior heating that is $1 \%$ of incident (dark green); and a $1+1-\mathrm{D}$ model with central heating that is $1 \%$ of incident and with $P_{n}=0.3$ (aqua). In order to limit the number of curves for ease of viewing, we present only a single purple curve (i.e., we do not display the evolution for when the heating is deposited at different pressure levels). Depositing the heat at 10 or 30 bars makes no discernible change to the purple curve. With 1-D models, if extra heating is deposited deep (e.g., 100 bars), the radius can be inflated more by heating in the atmosphere than by heating in the deep interior until late times (several Gyr); however, when night-side cooling is incorporated, extra heating in the deep interior inflates the radius more at all times later than a few Myr.

more (Showman \& Guillot 2002; Cooper \& Showman 2005; Langton \& Laughlin 2008; Dobbs-Dixon \& Lin 2008; Menou \& Rauscher 2009; Showman et al. 2009; Heng et al. 2011). If planets have large-scale dipolar magnetic fields (B), zonal winds will carry the free electrons across field lines, thereby inducing an electric field in response to the nonzero $\mathbf{u} \times \mathbf{B}$, as pointed out by both Perna et al. (2010a) and Batygin \& Stevenson (2010). The electric field will drive current loops that could close either in the atmosphere or in the interior. Here, we consider the consequences if currents ohmically dissipate power in the atmosphere.

Figure 9 shows five day-side atmospheric profiles (at fixed $T_{\text {eff }}$ ) for a planet with the incident stellar irradiation of HD 209458b. One profile is for a planet with no extra heating; the other four have heating profiles taken from Perna et al. (2010b), for 3-Gauss (G) magnetic fields (with and without associated drag on the flow) and for 10-G magnetic fields (with and without associated drag). Regions of the atmosphere that are convective are indicated in green. In the Perna et al. (2010b) models, stronger magnetic (and zero drag) fields lead to greater atmospheric heating.
As seen in Fig. 4, greater heating (at fixed $T_{\text {eff }}$ ) leads to greater interior entropy and larger radii. Figure 9]illustrates how atmospheric heating at constant $T_{\text {eff }}$ can modify the temperature-pressure profile to cause the atmosphere to match a higher entropy adiabat - the same process that leads to the radius inflation effect of atmospheric heating seen in Fig. 6 .

\subsection{Constraints on Ohmic Dissipation}

The ultimate power source of ohmic dissipation is stellar irradiation. Heating from the star drives winds, which, with a planetary-scale dipolar magnetic field and with free electrons in the atmosphere, lead to currents in the atmosphere that ohmically dissipate, dragging the winds and releasing heat in the atmosphere or in the convective interior. The power released by this process is bounded by the power incident on the planet.

The ohmic heating power per unit volume is $d P_{\text {ohm }} / d V=J^{2} / \sigma$, where $J$ is the current density and $\sigma$ is the electrical conductivity. For a dipolar magnetic field strength $B$, with zonal wind speed $u$, this can be 


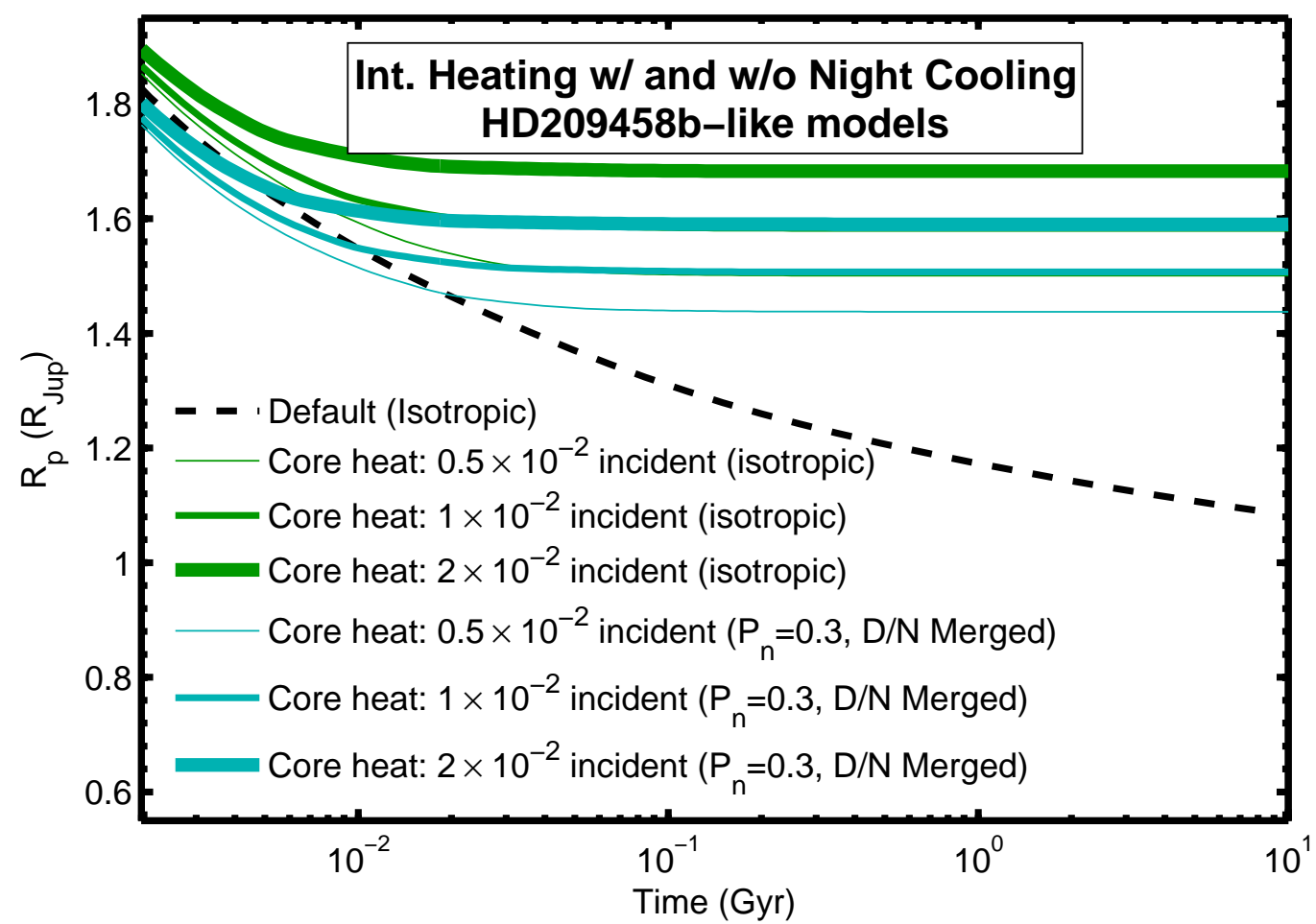

FIG. 7.- Radius evolution models of an HD 209458b-like planet with deep-interior heating, with and without night-side cooling. Seven models are presented: the default model, and six models with central heating $-0.5 \%, 1 \%$, and $2 \%$ of incident (thin, medium, and thick curves, respectively). Three of the models with central heating have isotropic boundary conditions (dark green), and three have 1+1-D boundary conditions with $P_{n}=0.3$. In this set of models, including night-side cooling with $P_{n}=0.3$ means that the model requires a factor of $\sim 2$ more interior heating to maintain the same radius.

expressed (Menou 2012a) as

$$
\frac{d P_{\mathrm{ohm}}}{d V}=\left(\frac{u}{c}\right)^{2} B^{2} \sigma,
$$

where $c$ is the speed of light. The electrical conductivity can be written

$$
\sigma=\frac{n_{e}}{n_{n}} \frac{e^{2} / m_{e}}{\langle A v\rangle}
$$

where, $n_{e}$ and $n_{n}$ are the number densities of free electrons and neutrals, respectively, $e$ is the electron charge, $m_{e}$ is the electron mass, $A$ is the electron cross section, and, per Draine et al. (1983), $\langle A v\rangle \sim$ $10^{-15}\left(128 k_{B} T / 9 \pi m_{e}\right)^{1 / 2}$. The ohmic heating density, therefore, is

$$
\begin{aligned}
\frac{d P_{\mathrm{ohm}}}{d V} \sim & 10^{3} \mathrm{erg} \mathrm{cm}^{-3} \mathrm{~s}^{-1}\left(\frac{u}{1 \mathrm{~km} / \mathrm{s}}\right)^{2}\left(\frac{B}{10 \mathrm{G}}\right)^{2} \\
& \times\left(\frac{Y_{e}}{10^{-4}}\right)\left(\frac{T}{10^{3} \mathrm{~K}}\right)^{-1 / 2}
\end{aligned}
$$

where the free-electron mixing ratio $Y_{e} \equiv n_{e} / n_{n}$. The scaling for $Y_{e}\left(10^{-4}\right)$ might seem strangely high, since at common conditions in hot Jupiter atmospheres most free electrons are contributed by singly ionized potassium and sodium, whose abundances (mixing ratios of $\sim 10^{-7}$ and $\sim 2 \times 10^{-6}$, respectively, if the composition is solar) are both much lower than $10^{-4}$. For the hottest hot Jupiters, however (e.g., WASP-12b and HAT-P-7b), ionization of hydrogen can contribute up to $\sim 10^{-4}$ in free-electron mixing ratio (see Fig. 10).

If the ohmic heating occurs over a solid angle $\pi$ of the planet's dayside, and extends vertically over $N$ scale heights $H$, then the integrated ohmic heating rate is

$$
\begin{aligned}
P_{\mathrm{ohm}} & \sim \pi R_{p}^{2} N H \frac{d P_{\mathrm{ohm}}}{d V} \\
& \sim\left(5 \times 10^{30} \frac{\mathrm{erg}}{\mathrm{s}}\right) N\left(\frac{R_{p}}{R_{J}}\right)^{2}\left(\frac{H}{350 \mathrm{~km}}\right) \\
& \left(\frac{u}{1 \mathrm{~km} / \mathrm{s}}\right)^{2}\left(\frac{B}{10 \mathrm{G}}\right)^{2}\left(\frac{Y_{e}}{10^{-4}}\right)\left(\frac{T}{10^{3} \mathrm{~K}}\right)^{-1 / 2} .
\end{aligned}
$$

The power incident on a planet a distance $a$ from a star of luminosity $L_{*}$ is

$$
P_{\text {in }} \sim 7 \times 10^{30} \mathrm{erg} \mathrm{s}^{-1}\left(\frac{L_{*}}{L_{\odot}}\right)\left(\frac{a}{0.01 \mathrm{AU}}\right)^{-2}\left(\frac{R_{p}}{R_{J}}\right)^{2} .
$$

Since the ohmic heating clearly cannot exceed the incident stellar heating, a comparison of equations (5) and (6) shows that there must be feedback between $B$ and $u$, whereby ohmic dissipation limits wind speeds. Since the heating power comes from the dissipation of wind velocities, this point is trivially true (Perna et al. 2010a, b; Batygin et al. 2011). Nevertheless, it is inter- 


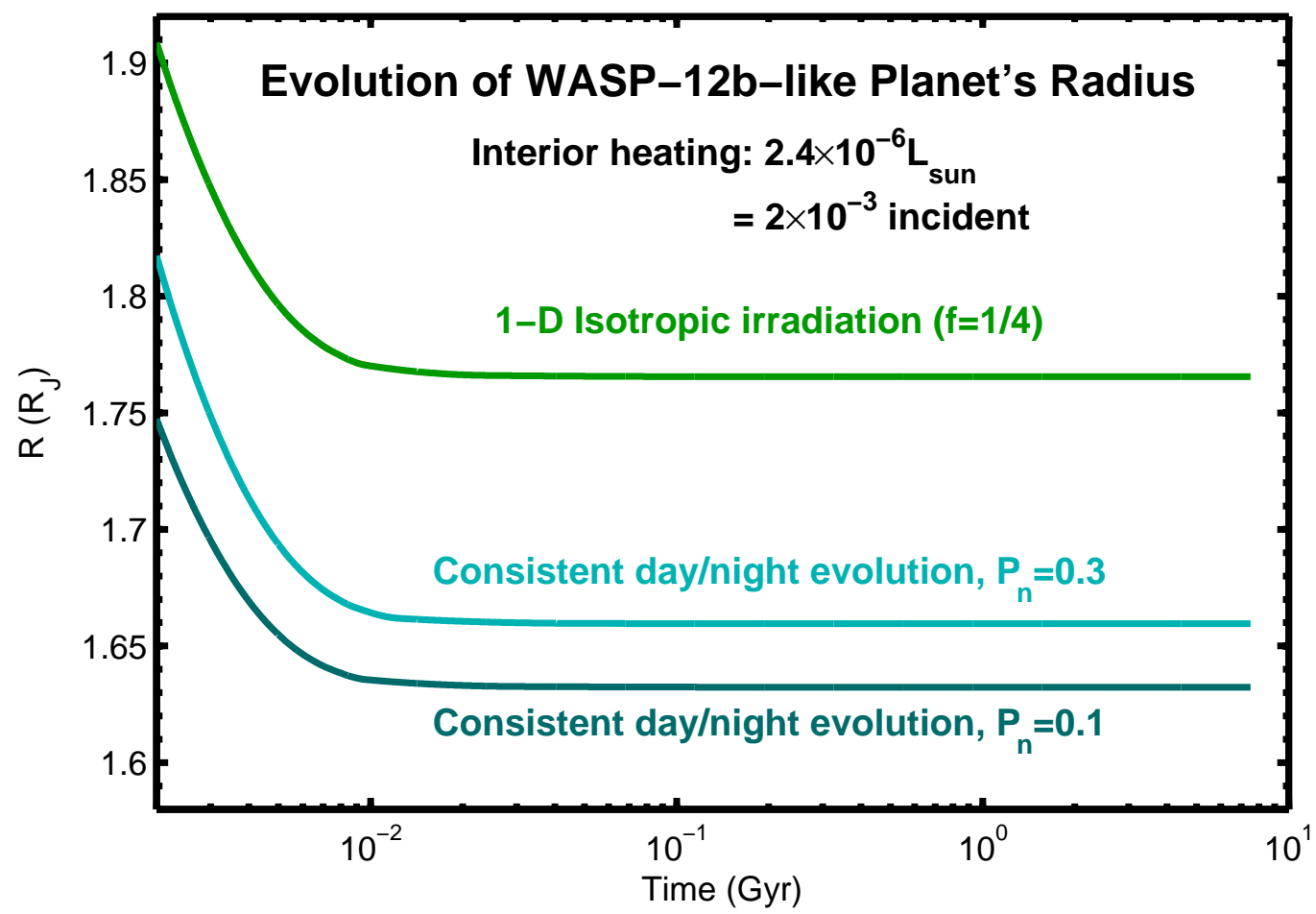

FIG. 8. - Radius of WASP-12b-like planet with interior heating. The evolution of radius is shown for a model planet the mass of WASP-12b $\left(1.39 M_{J}\right)$ for three different boundary conditions: a default $(f=1 / 4)$ model, and two models where the day and night sides are consistently coupled, with redistribution parameter $P_{n}$ set to 0.1 and to 0.3 . In all three cases, there is an extra interior power source equal to $2.4 \times 10^{-6} L_{\odot}$ (the value needed to maintain WASP-12b's radius, according to Ibgui et al. 2010). The planet cools more through the nightside than through the dayside (i.e., the effective temperature of the nightside is greater at equivalent entropy and surface gravity), which causes the models that incorporate night-side cooling to reach a lower asymptotic radius than is reached in the default $f=1 / 4$ case (which is essentially the model of Ibgui et al. 2010). Greater redistribution increases the night-side atmospheric temperatures, which slows the night-side cooling. Since the cooling is dominated by the night-side cooling, this also reduces the total cooling rate and leads to a slightly larger asymptotic radius at equivalent central heating power.

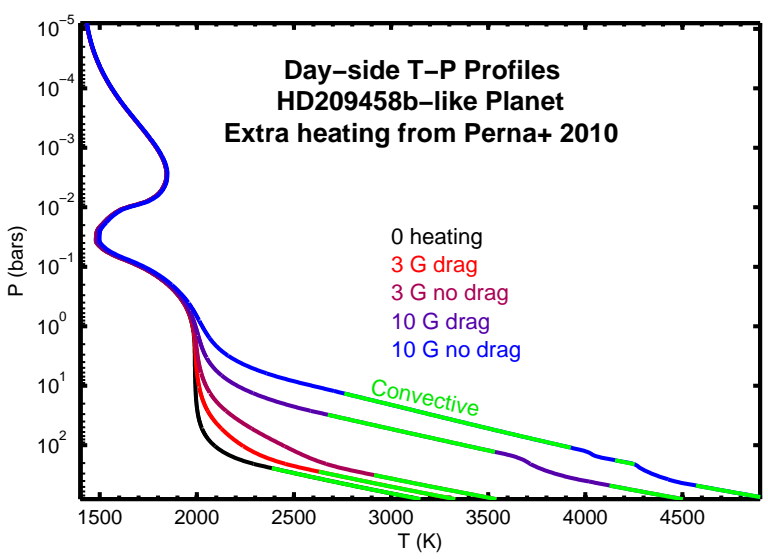

FIG. 9.- Temperature-pressure profiles in the presence of ohmic heating. Five atmospheric profiles are shown (at fixed $\left.T_{\text {eff }}\right)$ for a planet with the incident irradiation of HD 209458b. We adopt the atmospheric heating profiles presented in Perna et al. (2010b) for 3-G magnetic fields (with and without associated drag on the flow) and for 10-G magnetic fields (with and without associated drag). Stronger magnetic fields lead to greater atmospheric heating in the Perna et al. (2010b) models, and greater heating causes an atmosphere with a given effective temperature to have a base adiabat that is at higher entropy. Green regions are convective (and have uniform entropy) and other regions are radiative (and have positive radial entropy gradient).

esting to note that simple energy balance requires that, if the dipolar planetary magnetic field is of order $10 \mathrm{G}$, the hottest hot Jupiters cannot (over a large fraction of their day-side atmospheres) have zonal winds at the rkilometer-per-second level that is typically suggested by numerical simulations that do not include the magnetic drag effect, a conclusion that is also implied by the analysis of Menou (2012a).

Menou (2012a) suggests scaling relations for winds in ohmically-heated hot-Jupiter atmospheres. Without considering ohmic dissipation, the horizontal momentum equation leads to an order-of-magnitude estimate of (Showman et al. 2011)

$$
u^{2} \sim \frac{k \Delta T_{\text {horiz }}}{\mu m_{p}} \Delta \ln P,
$$

where $\Delta T_{\text {horiz }}$ is the day-night temperature contrast, $\mu$ is the mean molecular weight of the atmosphere in units of the proton mass $m_{p}$, and $\Delta \ln P$ is the number of scale heights above a base level of the atmosphere at which the day-side and night-side atmospheres have the same thermodynamic state. Note that equation (77) may be expressed as

$$
\begin{aligned}
u^{2} & \sim\left(c_{s_{\text {day }}}^{2}-c_{s_{\text {night }}^{2}}^{2}\right) \Delta \ln P, \quad \text { or }, \\
& \sim\left(c_{g_{\text {day }}}^{2}[2 \pi H]-c_{g_{\text {night }}^{2}}^{2}[2 \pi H]\right) \Delta \ln P,
\end{aligned}
$$


where the $c_{s}$ terms are the sound speeds on the day and night sides, and the $c_{g}[2 \pi H]$ terms are the gravity wave speeds for wavelength $2 \pi H$ on the day and night sides. For reasonable values of the day-night temperature difference, equations (7) and (8) yield zonal wind speeds of order the day-side sound speed, roughly a few kilometers per second $\left(\sim 2\left\{T_{\text {day }} / 1000 \mathrm{~K}\right\}^{1 / 2} \mathrm{~km} \mathrm{~s}^{-1}\right)$, which indicates that the magnetic drag term in equation (5) of Menou (2012a) plays an important role in limiting wind speeds if the magnetic field is of order several Gauss or more (Perna et al. 2010a, b). Note that most red-giant hot Jupiters (Spiegel \& Madhusudhan 2012) probably do not receive enough irradiation to have a high enough free-electron fraction to experience nonnegligible ohmic heating.

\subsection{Ionization and Heating in the Population of Known Hot Jupiters}

It is now possible to construct radiative-convective atmosphere models, guided by observations, of more than 20 transiting exoplanets. We have published models of over a dozen, including, in order of increasing incident stellar flux, HD 189733b (Burrows et al. 2008), HD 209458b (Knutson et al. 2008: Burrows et al. 2008), TrES-3 (Fressin et al. 2010), HAT-P-7b (Spiegel \& Burrows 2010), WASP18b (Machalek et al. 2010 submitted), and WASP-12b (Cowan et al. 2012). The radii of the members of this sextet, respectively, are $1.14 R_{J}, 1.35 R_{J}, 1.34 R_{J}$, $1.36 R_{J}, 1.11 R_{J}$, and $1.83 R_{J}$.

Figure 10 provides illustrative examples of the types of atmospheric structures that may be found across a range of hot Jupiter conditions. We present vertical profiles of (clockwise from upper left) atmospheric temperature, electron mixing ratio, conductivity, and ohmic heating for the six planets mentioned above, for which the incident flux varies from 0.48 to $9.10 \times 10^{9} \mathrm{erg} \mathrm{cm}^{-2} \mathrm{~s}^{-1}$, and $\log _{10}[\mathrm{~g}]$ (in cgs) ranges from 2.96 to 4.42 . The profiles assume day-side average conditions.

The top-left panel of Fig. 10 shows that three of these models - HD 209458b, HAT-P-7b, and WASP$12 \mathrm{~b}$ - have thermal inversions in their upper atmospheres, although that of WASP-12b is somewhat disputed (see Madhusudhan et al. 2011, Cowan et al. 2012, and Crossfield et al.|2013 for further discussion). All one-dimensional models of sufficiently highly irradiated planets have deep, nearly isothermal, layers, extending more than two decades in pressure down from the photosphere at $\sim 1$ bar. In general, the temperature of the isothermal layer scales with the strength of the irradiation roughly in proportion to $F_{0}^{1 / 4}$, where $F_{0}$ is the incident flux at the substellar point (Hubeny et al. 2003). However, the presence of a thermal inversion tends to heat the upper atmosphere and cool the lower atmosphere relative to the profile that would obtain without an inversion, as is clear from examining the relative temperature profiles of, e.g., WASP-18b and WASP-12b. The electron mixing ratio is calculated via Saha equations, assuming solar composition of the elements. The conductivity is calculated with equation (3), found in Menou (2012a).

In order to produce the heating profile plot in the lower right panel of Fig. 10, we use equation (4) to calculate the ohmic heating density, and vertically integrate this density times $\pi R_{p}^{2}$ from the outside of the planet inward. The abscissa, then, is the ratio of the cumulative ohmic power (from the outside of the planet down to a given ordinate) to the incident stellar power. Note that the ratio of the full integral of the ohmic power to the incident irradiation is the efficiency factor $\varepsilon$ referred to in $\$ 5.4$ below. The temperature and electron mixing ratio profiles are calculated from the model atmospheres, and the zonal wind speed $u$ and magnetic field strength $B$ are fixed at values motivated by previous studies in the literature. We present two heating profiles for each planet. The solid curve assumes zonal wind speeds of $1 \mathrm{~km} \mathrm{~s}^{-1}$ throughout the whole atmosphere, where this speed is motivated by the results of a variety of circulation models in the literature and by the simple scalings in equations (7) and (8). The dashed curve assumes that the zonal wind speed is prograde (relative to the corotating frame) by $1 \mathrm{~km} \mathrm{~s}^{-1}$ at pressures lower than $10^{-4}$ bars, stationary $\left(0 \mathrm{~km} \mathrm{~s}^{-1}\right)$ at pressures greater than 300 bars, and varies linearly with altitude in between. The magnetic fields are assumed to be $3 \mathrm{G}$ for all planets. Note that increasing $B$ from $3 \mathrm{G}$ to $10 \mathrm{G}$ increases the heating by roughly an order of magnitude, which results in the cumulative ohmic power being greater than the incident stellar power for the models of HAT-P-7b and WASP-12b if the winds are $\sim 1 \mathrm{~km} \mathrm{~s}^{-1}$ over a significant portion of the day-side atmosphere. In truth, the wind speeds would depend on the magnetic field strength, which itself depends on hydrodynamic motions in the deep interior and in the atmosphere that, presumably, lead to a magnetic dynamo. Numerous works have attempted to simulate or calculate aspects of this feedback, with varying levels of sophistication, although none of these works captures the full process (Perna et al. 2010a, b; Batvgin et al. 2011; Menou 2012a; Rauscher \& Menou 2013; Wu \& Lithwick 2013). The profiles presented here should be taken not as quantitative predictions, but rather as qualitative guides to the vertical variation in heating that might obtain in hot Jupiter atmospheres at a range of levels of irradiation. These profiles may be scaled with $u^{2} B^{2}$ for different assumptions of wind speeds and magnetic field strengths. Clearly, the largest contribution to the degree of ohmic heating is the local conductivity, which varies by $\sim 8$ orders of magnitude across different altitudes and different planets.

The ionization depends very sensitively on temperature. On the nightsides of these planets (calculated, but not shown), the temperatures are cool enough that electron mixing ratios and, therefore, electrical conductivities, are much lower than on the daysides. The night-side conductivities are all $\lesssim 10^{4} \mathrm{~s}^{-1}$, render- 
ing any implied heating rate too low $\left(<10^{-4}\right.$ of the incident stellar power) to have a significant effect on planet radius.

Our one-dimensional atmosphere models of the day and night sides of highly irradiated gas-giant planets suggest that the nightsides might have very low free-electron fractions and experience negligible ohmic heating. The daysides of the hottest hot Jupiters, however, should be highly enough ionized that that they might experience significant ohmic heating if wind speeds are as high as dynamical models have suggested and if the magnetic field strengths are as great as Jupiter's 4-Gauss field (Russell 1993; Christensen et al. 2009).

\subsection{Planetary Radii Are Generally Stable Against Runaway Expansion Due to Ohmic Heating}

In order to investigate the stability of a planet's radius, we consider how an equilibrium situation responds to a perturbation. Define $R_{p}^{0}$ as the equilibrium radius. That is, at $R_{p}=R_{p}^{0}$, heating balances cooling and $d R_{p} / d t=0$. Let $s$ be the dimensionless specific entropy per baryon - in a fully convective, approximately isentropic object, essentially the total entropy $\mathcal{S}$ divided by the product of Boltzmann's constant $k_{B}$ with the total number of baryons $M_{p} / m_{p}$ : $s=\mathcal{S} /\left(k_{B} M_{p} / m_{p}\right)$.

Note that, in a differential mass element $d m$, a change in heat $d q$ corresponds to a change in entropy via $d q=k_{B} T(d \mathcal{S} / d t) d t=k_{B} N_{A} T(d s / d t) d m d t$, where the operator $d$ indicates an incomplete differential and $N_{A}$ is Avogadro's number. So, the mass-integrated change in heat $\Varangle Q$ may be expressed as:

$$
\varpi Q=\left(\int_{\text {Mass }} k_{B} N_{A} T \frac{d s}{d t} d m\right) d t=L_{\text {net }- \text { in }} d t=-L d t,
$$

where $L_{\text {net-in }}=-L$ is the net inward (heating) power, which is the negative of $L$, the net outward (cooling) luminosity. The net luminosity $L=C-H$, where $C$ is the outward luminosity and $H$ is the inward luminosity (and, at $R_{p}^{0}, C\left[R_{p}^{0}\right]=H\left[R_{p}^{0}\right]$ ).

These are the ingredients needed to evaluate $d R_{p} / d t$ - i.e., $\left(d R_{p} / d s\right)(d s / d t)$ - for a given perturbation $\Delta R_{p}$ from $R_{p}^{0}$. If we define $\delta_{R_{p}} \equiv \Delta R_{p} / R_{p}^{0}$, we find that, unsurprisingly,

$$
\dot{\delta}_{R_{p}} \equiv \frac{d \delta_{R_{p}}}{d t} \sim-\frac{\delta_{R_{p}}}{\tau},
$$

where the relaxation timescale $\tau$ may be written

$$
\tau=\frac{\frac{1}{m_{p}} \int k_{B} T d m}{\frac{d R}{d s} \frac{d L}{d R_{p}}} \sim \frac{\widetilde{E}}{L} .
$$

Here, $\widetilde{E}$ is of order the product of the number of particles $\left(\sim M_{p} / m_{p}\right)$ with the mass-weighted average of $k_{B} T\left(\sim M_{p}^{-1} \int k_{B} T d m\right)$. The form of $\tau$ makes clear that it is essentially the Kelvin-Helmholtz timescale, which is the natural timescale on which an object responds to thermal perturbations. Importantly, a perturbation relaxes to equilibrium since the coefficient of $\delta_{R_{p}}$ in the relation $\dot{\delta}_{R_{p}} \propto \delta_{R_{p}}$ is negative so long as $d L / d R_{p}$ is positive.

Figure 11 shows illustrative curves for heating power versus cooling power for models of an irradiated hot Jupiter planet (similar to HD 209458b). Intersections between the heating curves and the cooling curves represent equilibrium radii (" $R_{P}^{0}$ ") where the cooling balances the heating. The upshot of the stability argument just presented is evident in this figure, because at all equilibria the slope of the cooling curve is greater than the slope of the heating curve, indicating that a small positive perturbation to the radius will lead to cooling exceeding heating and, therefore, to the radius shrinking back to the equilibrium. This indicates that, in these models, there is no purely thermal runaway instability in radius (for $\varepsilon \lesssim 10 \%$ ). This result is in contrast to the suggestion in Batygin et al. (2011) that ohmic heating can lead to a thermal instability that culminates in complete evaporation of a planet. When a planet's radius becomes large enough, however, a runaway Roche-lobe overflow process can still occur that might initially be triggered by ohmic heating.

\section{CONCLUSIONS}

We have presented an analysis of various subtle, but important, physical effects that can influence the radii of hot Jupiters. To explain the anomalously large radii of an interesting subset of this class, some have invoked the deposition of thermal power either in planets' deep, convective interiors or at various levels in their radiative atmospheres. To explore the consequences of such heating on the radii and radius evolution of hot Jupiters, we generated self-consistent atmosphere-planet evolutionary models and found that the evolution of highly irradiated gas-giant planets depends sensitively on where the energy is deposited. If "extra" power goes into heating the upper atmosphere, via an extra thermal-inversioncausing optical absorber, this (slightly) increases the evolutionary cooling rate and results in slightly smaller radii. If the additional power is deposited more deeply in the radiative atmosphere (e.g., via ohmic heating), the interior cooling rate decreases, with the result that the planet's radius is larger at a given age. The radiusboosting effect of a given amount of power increases with the depth at which that power is delivered in the atmosphere. However, the asymptotic radius in the limit of infinite age is not changed. In other words, atmospheric heating might be able to produce a large inflating effect, but one that does not last forever. In contrast, the same power deposited deep in the convective interior of the planet always has a larger asymptotic radius than if the same power is delivered anywhere in the radiative zone.

Importantly, cooling through the (cooler) nightside of a tidally locked hot Jupiter, or through its (cooler) 

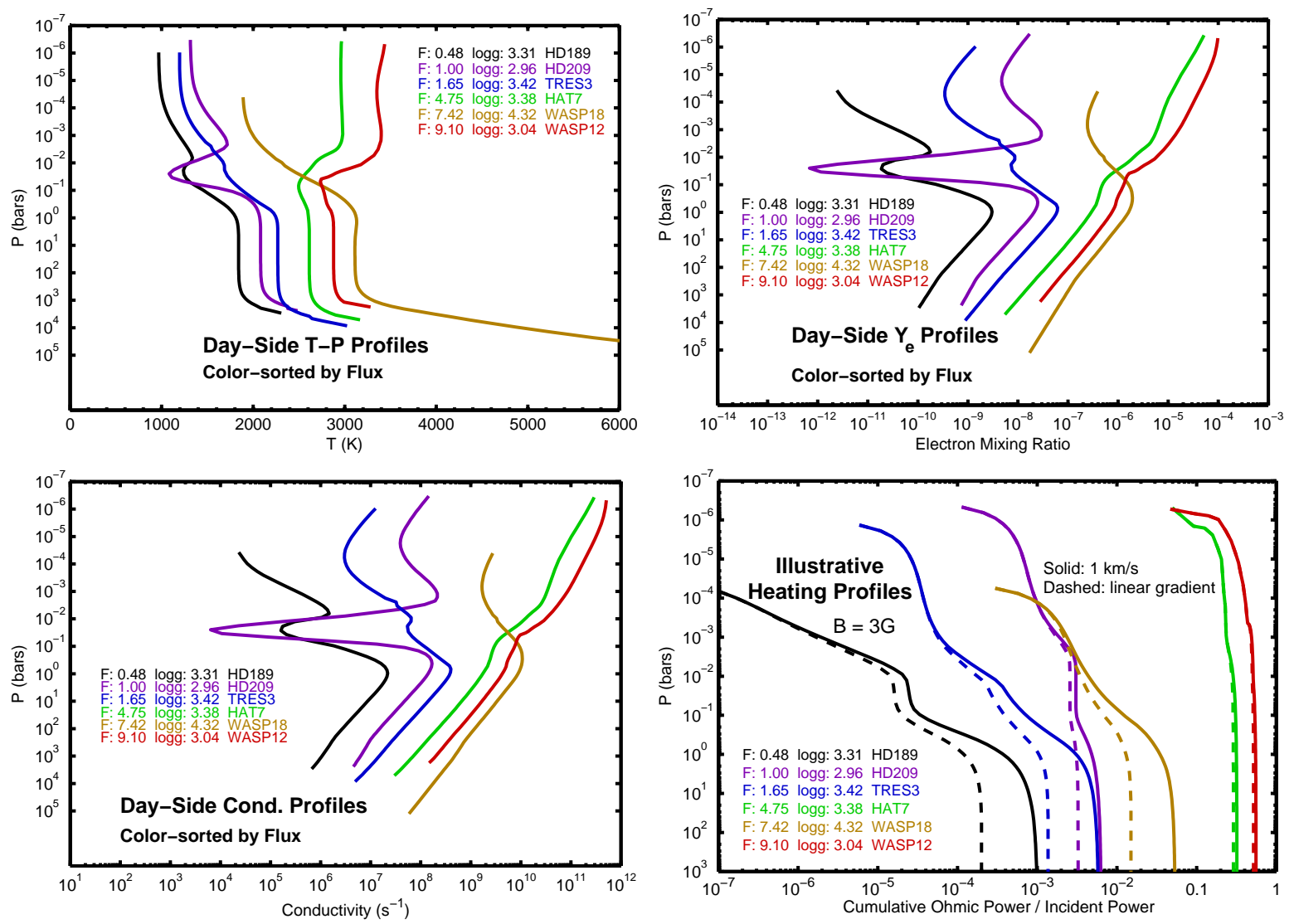

FIG. 10.- Profiles of properties of day-side atmospheres of transiting exoplanets. Each panel shows pressure as the ordinate (decreasing upward on a logarithmic scale; i.e., altitude increases upward approximately linearly) and some other atmospheric property as the abscissa. The profiles are color-sorted by incident flux, and the legend in each panel indicates incident flux ("F:") in units of $10^{9} \mathrm{erg} \mathrm{cm}^{-2} \mathrm{~s}^{-1}$ and the base-10 logarithm of surface gravity ("logg:") in dynes per gram. Top left: Temperature profiles are shown, including some corresponding to thermally-inverted atmospheres (e.g., HD 209458b, WASP-18b, and WASP-12b - although see Madhusudhan et al. 2011). More highly irradiated planets tend to have hotter isothermal layers between $\sim 1$ and $\sim 300$ bars. Top right: Electron mixing ratios are derived from pressure and temperature according to Saha equations. Most of the free electrons in the upper atmosphere come from singly ionized sodium and potassium. All atmospheres shown are at most weakly ionized (the maximum ionization shown is $\sim 10^{-4}$, for a model of WASP-12b), but, importantly, the ionization is nonzero. More highly irradiated planets have greater partial ionization. (High in the atmosphere - at microbar pressures - there can be a nonnegligible photoionization component to the free electron fraction.) Bottom left: Conductivity profiles are derived from the free electron number density. Bottom right: Heating profiles are derived from the conductivity, with assumptions about the magnetic field strength (3 Gauss) and wind speeds (solid: $1.0 \mathrm{~km} / \mathrm{s}$; dashed: $1.0 \mathrm{~km} / \mathrm{s}$ at pressures lower than $10^{-4} \mathrm{bars}, 0.0 \mathrm{~km} / \mathrm{s}$ at pressures greater than 300 bars, and varying linearly with altitude in between).

poles, increases the rate of interior cooling and radius shrinkage relative to isotropic models. As a result, the radius-inflating influence of atmospheric heating is reduced in a 1+1-D model that consistently treats day/night redistribution of heat and cooling through the nightside. This makes it difficult to significantly increase the radius of a hot Jupiter via atmospheric heating alone.

In addition, we find a limit on the product of zonal wind speed and magnetic field strength in hot Jupiter atmospheres that have significant ionization. Specifically, the most highly irradiated planets cannot have $u B \gtrsim 10 \mathrm{~km} \mathrm{~s}^{-1}$ Gauss over a large fraction of their daysides, where $u$ is the zonal wind speed and $B$ is the dipolar magnetic field strength in the atmosphere.

The vexing problem of the inflated radii of some hot Jupiters has persisted for more than a decade. It has long been appreciated that intense irradiation can slow the evolutionary cooling of these objects, whatever other processes are invoked to explain the anomaly. Here, however, we have shown that coupling the day-side cooling with that through the nightside or the polar regions largely undoes the reduced interior cooling effect of the irradiating flux. An extra power source may, then, be needed in their deep interiors, not merely in their atmospheres, to explain most inflated, multi-billion-year-old planets. Moreover, the degree of extra power that must be deposited in the deep interior would seem to be greater than has been inferred in those many studies using isotropically cooling models.

We thank Ivan Hubeny, Kristen Menou, Adam Showman, Jano Budaj, Yanqin Wu, and Greg Novak for useful discussions. The authors acknowledge support in part under NASA ATP grant NNX07AG80G, HST grants HST-GO-12181.04-A and 


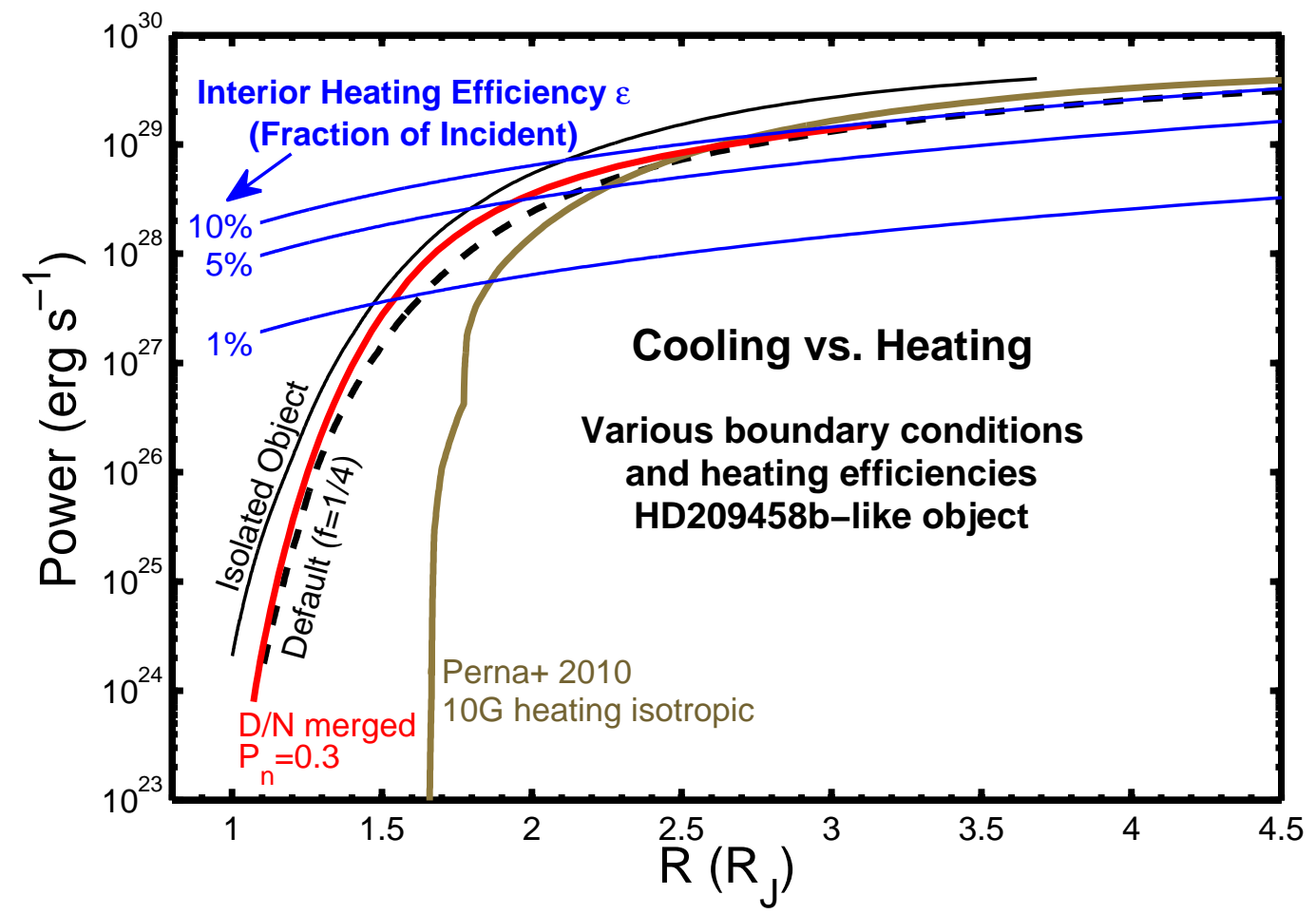

FIG. 11. - Heating and cooling rates vs. total radius for irradiated models of an HD 209458b-like planet. The heating curves (blue) show the heating power as a function of planetary radius, for three different assumed (constant) efficiencies of converting intercepted power to interior heating: $1 \%, 5 \%$, and $10 \%$. The cooling curves represent $4 \pi R_{p}^{2} \sigma T_{\text {eff }}^{4}$, for four different cases: an isolated object (thin black) cooling with the boundary condition of Burrows et al. (1997); the 1-d $f=1 / 4$ boundary condition (dashed black) presented in Figs. 3 and 5 7 7 the 1+1-D boundary condition with $P_{n}=0.3$ (red) presented in Figs. [5 and 6] and a 1-D boundary condition assuming an atmospheric heating profile corresponding to 10 Gauss with drag from Perna et al. (2010b). For efficiencies less than $\sim 10 \%$, all cooling curves cross a heating curve at some (radius,power) pair, meaning that there is no purely thermal runaway instability in radius because the cooling power at some point balances (or exceeds) the heating power, although Roche-lobe overflow might occur when the radius becomes large enough, for planets that are particularly close-in.

HST-GO-12314.03-A, and JPL/Spitzer Agreements $1417122,1348668,1371432$, and 1377197. DSS gratefully acknowledges support from NSF grant AST-
0807444 and the Keck Fellowship, and the Friends of the Institute.

\section{REFERENCES}

Anderson, D. R., Smith, A. M. S., Lanotte, A. A., Barman, T. S., Collier Cameron, A., Campo, C. J., Gillon, M., Harrington, J., Hellier, C., Maxted, P. F. L., Queloz, D., Triaud, A. H. M. J., \& Wheatley, P. J. 2011, MNRAS, 416, 2108

Arras, P. \& Socrates, A. 2009a, ArXiv e-prints arXiv:0912.2318

- 2009b, ArXiv e-prints arXiv:0901.0735

-. 2010, ApJ, 714, 1

Baraffe, I., Alibert, Y., Chabrier, G., \& Benz, W. 2006, A\&A, 450, 1221

Baraffe, I., Chabrier, G., \& Barman, T. 2008, A\&A, 482, 315

- 2010, Reports on Progress in Physics, 73, 016901

Baraffe, I., Chabrier, G., Barman, T. S., Allard, F., \& Hauschildt, P. H. 2003, A\&A, 402, 701

Baraffe, I., Chabrier, G., Barman, T. S., Selsis, F., Allard, F., \& Hauschildt, P. H. 2005, A\&A, 436, L47

Baraffe, I., Selsis, F., Chabrier, G., Barman, T. S., Allard, F., Hauschildt, P. H. \& Lammer, H. 2004, A\& A, 419, L13

Batygin, K. \& Stevenson, D. J. 2010, ApJ, 714, L238

Batygin, K., Stevenson, D. J., \& Bodenheimer, P. H. 2011, ApJ, 738, 1

Bodenheimer, P., Laughlin, G., \& Lin, D. N. C. 2003, ApJ, 592,555

Bodenheimer, P., Lin, D. N. C., \& Mardling, R. A. 2001, ApJ, 548,466
Boss, A. P. 1995, Science, 267, 360

Budaj, J. 2011, AJ, 141, 59

Budaj, J., Hubeny, I., \& Burrows, A. 2012, A\&A, 537, A115

Burrows, A., Budaj, J., \& Hubeny, I. 2008, ApJ, 678, 1436

Burrows, A., Guillot, T., Hubbard, W. B., Marley, M. S.,

Saumon, D., Lunine, J. I., \& Sudarsky, D. 2000, ApJ, 534, L97

Burrows, A., Heng, K., \& Nampaisarn, T. 2011, ApJ, 736, 47

Burrows, A., Hubbard, W. B., \& Lunine, J. I. 1994, in Astronomical Society of the Pacific Conference Series,

Vol. 64, Cool Stars, Stellar Systems, and the Sun, ed. J.-P. Caillault, 528-+

Burrows, A., Hubbard, W. B., Lunine, J. I., \& Liebert, J. 2001, Reviews of Modern Physics, 73, 719

Burrows, A., Hubeny, I., Budaj, J., \& Hubbard, W. B. 2007a, ApJ, 661, 502

Burrows, A., Hubeny, I., Budaj, J., Knutson, H. A., \& Charbonneau, D. 2007b, ApJ, 668, L171

Burrows, A., Hubeny, I., Hubbard, W. B., Sudarsky, D., \& Fortney, J. J. 2004, ApJ, 610, L53

Burrows, A. \& Liebert, J. 1993, Reviews of Modern Physics, 65,301

Burrows, A., Marley, M., Hubbard, W. B., Lunine, J. I., Guillot, T., Saumon, D., Freedman, R., Sudarsky, D., \& Sharp, C. 1997, ApJ, 491, 856 
Burrows, A., Rauscher, E., Spiegel, D. S., \& Menou, K. 2010, ApJ, 719, 341

Burrows, A., Sudarsky, D., \& Hubbard, W. B. 2003, ApJ, 594, 545

Burrows, A., Sudarsky, D., \& Hubeny, I. 2006, ApJ, 650, 1140 Chabrier, G. \& Baraffe, I. 2007, ApJ, 661, L81

Chabrier, G., Barman, T., Baraffe, I., Allard, F., \& Hauschildt, P. H. 2004, ApJ, 603, L53

Chan, T., Ingemyr, M., Winn, J. N., Holman, M. J., Sanchis-Ojeda, R., Esquerdo, G., \& Everett, M. 2011, AJ, 141,179

Charbonneau, D., Brown, T. M., Latham, D. W., \& Mayor, M. 2000, ApJ, 529, L45

Christensen, U. R., Holzwarth, V., \& Reiners, A. 2009, Nature, 457,167

Cooper, C. S. \& Showman, A. P. 2005, ApJ, 629, L45

Cowan, N. B. \& Agol, E. 2011, ApJ, 729, 54

Cowan, N. B., Machalek, P., Croll, B., Shekhtman, L. M., Burrows, A., Deming, D., Greene, T., \& Hora, J. L. 2012, ApJ, 747, 82

Crossfield, I. J. M., Barman, T., Hansen, B. M. S., Tanaka, I., \& Kodama, T. 2013, ArXiv e-prints

Demory, B.-O. \& Seager, S. 2011, ApJS, 197, 12

Dobbs-Dixon, I. \& Lin, D. N. C. 2008, ApJ, 673, 513

Draine, B. T., Roberge, W. G., \& Dalgarno, A. 1983, ApJ, 264,485

Fortney, J. J. \& Hubbard, W. B. 2004, ApJ, 608, 1039

Fortney, J. J., Ikoma, M., Nettelmann, N., Guillot, T., \& Marley, M. S. 2011, ApJ, 729, 32

Fortney, J. J., Lodders, K., Marley, M. S., \& Freedman, R. S. 2008a, ApJ, 678, 1419

Fortney, J. J. \& Marley, M. S. 2007, ApJ, 666, L45

Fortney, J. J., Marley, M. S., Saumon, D., \& Lodders, K. 2008b, ApJ, 683, 1104

Fressin, F., Knutson, H. A., Charbonneau, D., O'Donovan, F. T., Burrows, A., Deming, D., Mandushev, G., \& Spiegel, D. 2010, ApJ, 711, 374

Goodman, J. \& Lackner, C. 2009, ApJ, 696, 2054

Gu, P.-G., Lin, D. N. C., \& Bodenheimer, P. H. 2003, ApJ, 588,509

Guillot, T. 2010, A\&A, 520, A27+

Guillot, T., Burrows, A., Hubbard, W. B., Lunine, J. I., \& Saumon, D. 1996, ApJ, 459, L35+

Guillot, T., Santos, N. C., Pont, F., Iro, N., Melo, C., \& Ribas, I. 2006, A\&A, 453, L21

Guillot, T. \& Showman, A. P. 2002, A\&A, 385, 156

Hansen, B. M. S. 2008, ApJS, 179, 484

Hartman, J. D., Bakos, G. Á., Torres, G., Latham, D. W. Kovács, G., Béky, B., Quinn, S. N., Mazeh, T., Shporer, A., Marcy, G. W., Howard, A. W., Fischer, D. A., Johnson, J. A., Esquerdo, G. A., Noyes, R. W., Sasselov, D. D. Stefanik, R. P., Fernandez, J. M., Szklenár, T., Lázár, J., Papp, I., \& Sári, P. 2011, ApJ, 742, 59

Hebb, L., Collier-Cameron, A., Loeillet, B., Pollacco, D. Hébrard, G., Street, R. A., Bouchy, F., Stempels, H. C. Moutou, C., Simpson, E., Udry, S., Joshi, Y. C., West, R. G., Skillen, I., Wilson, D. M., McDonald, I., Gibson, N. P., Aigrain, S., Anderson, D. R., Benn, C. R., Christian, D. J., Enoch, B., Haswell, C. A., Hellier, C., Horne, K., Irwin, J., Lister, T. A., Maxted, P., Mayor, M., Norton, A. J., Parley, N., Pont, F., Queloz, D., Smalley, B., \& Wheatley, P. J. 2009, ApJ, 693, 1920

Heng, K., Menou, K., \& Phillipps, P. J. 2011, MNRAS, 413, 2380

Henry, G. W., Marcy, G. W., Butler, R. P., \& Vogt, S. S. 2000, ApJ, 529, L41
Howard, A. W., Marcy, G. W., Bryson, S. T., Jenkins, J. M., Rowe, J. F., Batalha, N. M., Borucki, W. J., Koch, D. G., Dunham, E. W., Gautier, III, T. N., Van Cleve, J., Cochran, W. D., Latham, D. W., Lissauer, J. J., Torres, G., Brown, T. M., Gilliland, R. L., Buchhave, L. A., Caldwell, D. A., Christensen-Dalsgaard, J., Ciardi, D., Fressin, F., Haas, M. R., Howell, S. B., Kjeldsen, H., Seager, S., Rogers, L., Sasselov, D. D., Steffen, J. H., Basri, G. S., Charbonneau, D., Christiansen, J., Clarke, B., Dupree, A., Fabrycky, D. C., Fischer, D. A., Ford, E. B., Fortney, J. J., Tarter, J., Girouard, F. R., Holman, M. J., Johnson, J. A., Klaus, T. C., Machalek, P., Moorhead, A. V., Morehead, R. C Ragozzine, D., Tenenbaum, P., Twicken, J. D., Quinn, S. N., Isaacson, H., Shporer, A., Lucas, P. W., Walkowicz, L. M., Welsh, W. F., Boss, A., Devore, E., Gould, A., Smith, J. C., Morris, R. L., Prsa, A., Morton, T. D., Still, M., Thompson, S. E., Mullally, F., Endl, M., \& MacQueen, P. J. 2012, ApJS, 201, 15

Huang, X. \& Cumming, A. 2012, ApJ, 757, 47

Hubeny, I. 1988, Computer Physics Communications, 52, 103

Hubeny, I., Burrows, A., \& Sudarsky, D. 2003, ApJ, 594, 1011

Hubeny, I. \& Lanz, T. 1995, ApJ, 439, 875

Ibgui, L. \& Burrows, A. 2009, ApJ, 700, 1921

Ibgui, L., Burrows, A., \& Spiegel, D. S. 2010, ApJ, 713, 751

Ibgui, L., Spiegel, D. S., \& Burrows, A. 2011, ApJ, 727, 75

Jackson, B., Greenberg, R., \& Barnes, R. 2008, ApJ, 681, 1631

Knutson, H. A., Charbonneau, D., Allen, L. E., Burrows, A., \& Megeath, S. T. 2008, ApJ, 673, 526

Knutson, H. A., Howard, A. W., \& Isaacson, H. 2010, ApJ, 720,1569

Langton, J. \& Laughlin, G. 2008, ApJ, 674, 1106

Laughlin, G., Crismani, M., \& Adams, F. C. 2011, ApJ, 729, L7

Laughlin, G., Wolf, A., Vanmunster, T., Bodenheimer, P., Fischer, D., Marcy, G., Butler, P., \& Vogt, S. 2005, ApJ, 621,1072

Leconte, J. \& Chabrier, G. 2012, A\&A, 540, A20

Leconte, J., Chabrier, G., Baraffe, I., \& Levrard, B. 2010, A\&A, 516, A64

Lissauer, J. J. 1995, Icarus, 114, 217

Liu, X., Burrows, A., \& Ibgui, L. 2008, ApJ, 687, 1191

Lubow, S. H., Tout, C. A., \& Livio, M. 1997, ApJ, 484, 866

Machalek, P., Greene, T., Harrington, J., Burrows, A., \&

Hubeny, I. 2010 submitted, ApJ

Madhusudhan, N. 2012, ApJ, 758, 36

Madhusudhan, N., Harrington, J., Stevenson, K. B., Nymeyer,

S., Campo, C. J., Wheatley, P. J., Deming, D., Blecic, J., Hardy, R. A., Lust, N. B., Anderson, D. R.,

Collier-Cameron, A., Britt, C. B. T., Bowman, W. C., Hebb, L., Hellier, C., Maxted, P. F. L., Pollacco, D., \& West, R. G. 2011, Nature, 469, 64

Madhusudhan, N. \& Seager, S. 2009, ApJ, 707, 24

—. 2010, ApJ, 725, 261

Marcy, G. W. \& Butler, R. P. 1996, ApJ, 464, L147+

Marleau, G.-D. \& Cumming, A. 2013, ArXiv e-prints arXiv: 1302.1517

Marley, M. S., Fortney, J. J., Hubickyj, O., Bodenheimer, P., \& Lissauer, J. J. 2007, ApJ, 655, 541

Mayor, M. \& Queloz, D. 1995, Nature, 378, 355

Menou, K. 2012a, ApJ, 745, 138

-. 2012b, ApJ, 754, L9

Menou, K. \& Rauscher, E. 2009, ApJ, 700, 887

Miller, N., Fortney, J. J., \& Jackson, B. 2009, ApJ, 702, 1413

Ogilvie, G. I. \& Lin, D. N. C. 2007, ApJ, 661, 1180

Parmentier, V., Showman, A. P., \& Lian, Y. 2013, ArXiv e-prints arXiv: 1301.4522

Paxton, B., Cantiello, M., Arras, P., Bildsten, L., Brown, E. F., Dotter, A., Mankovich, C., Montgomery, M. H., Stello, D., Timmes, F. X., \& Townsend, R. 2013, ArXiv e-prints arXiv: 1301.0319

Perna, R., Menou, K., \& Rauscher, E. 2010a, ApJ, 719, 1421

-. 2010b, ApJ, 724, 313

Rauscher, E. \& Menou, K. 2013, ApJ, 764, 103

Rein, H. 2012, ArXiv e-prints arXiv:1211.7121 
Russell, C. T. 1993, Reports on Progress in Physics, 56, 687 Schneider, J., Dedieu, C., Le Sidaner, P., Savalle, R., \& Zolotukhin, I. 2011, A\& A, 532, A79

Sharp, C. M. \& Burrows, A. 2007, ApJS, 168, 140

Showman, A. P., Cho, J. Y.-K., \& Menou, K. Atmospheric Circulation of Exoplanets, ed. S. Seager, 471-516

Showman, A. P., Fortney, J. J., Lian, Y., Marley, M. S., Freedman, R. S., Knutson, H. A., \& Charbonneau, D. 2009, ApJ, 699, 564

Showman, A. P. \& Guillot, T. 2002, A\&A, 385, 166

Socrates, A. 2013, ArXiv e-prints arXiv:1304.4121

Spiegel, D. S. \& Burrows, A. 2010, ApJ, 722, 871

-. 2012, ApJ, 745, 174
Spiegel, D. S., Burrows, A., \& Milsom, J. A. 2011, ApJ, 727, 57 Spiegel, D. S. \& Madhusudhan, N. 2012, ApJ, 756, 132

Spiegel, D. S., Silverio, K., \& Burrows, A. 2009, ApJ, 699, 1487

Struve, O. 1952, The Observatory, 72, 199

Wright, J. T., Fakhouri, O., Marcy, G. W., Han, E., Feng, Y., Johnson, J. A., Howard, A. W., Fischer, D. A., Valenti,

J. A., Anderson, J., \& Piskunov, N. 2011, PASP, 123, 412

Wu, Y. 2005a, ApJ, 635, 674

- 2005b, ApJ, 635, 688

Wu, Y. \& Lithwick, Y. 2013, ApJ, 763, 13

Zapolsky, H. S. \& Salpeter, E. E. 1969, ApJ, 158, 809 